\title{
Endovascular repair of thoracoabdominal aortic aneurysms using fenestrated and branched endografts
}

\author{
Gustavo S. Oderich, MD, ${ }^{\mathrm{a}}$ Mauricio Ribeiro, $\mathrm{MD}, \mathrm{PhD},{ }^{\mathrm{a}, \mathrm{c}}$ Leonardo Reis de Souza, MD, ${ }^{\mathrm{a}}$ Jan Hofer, $\mathrm{RN},{ }^{\mathrm{a}}$ \\ Jean Wigham, RN, ${ }^{\mathrm{a}}$ and Stephen Cha, $\mathrm{MS}^{\mathrm{b}}$
}

\begin{abstract}
Purpose: The study purpose was to review the outcomes of patients treated for thoracoabdominal aortic aneurysms using endovascular repair with fenestrated and branched stent-grafts in a single center.

Methods: We reviewed the clinical data of the first 185 consecutive patients (134 male; mean age, $75 \pm 7$ years) treated for thoracoabdominal aortic aneurysms using fenestrated and branched stent-grafts. Graft design evolved from physicianmodified endografts (2007-2013) to off-the-shelf or patient-specific manufactured devices in patients enrolled in a prospective physician-sponsored investigational device exemption protocol (NCT 1937949 and 2089607). Outcomes were reported for extent IV and extent I to III thoracoabdominal aortic aneurysms, including 30-day mortality, major adverse events, patient survival, primary target vessel patency, and reintervention.
\end{abstract}

Results: A total of 112 patients $(60 \%)$ ) were treated for extent IV thoracoabdominal aortic aneurysms, and 73 patients $(40 \%)$ were treated for extent I to III thoracoabdominal aortic aneurysms. Demographics and cardiovascular risk factors were similar in both groups. A total of 687 renal-mesenteric arteries (3.7 vessels/patient) were targeted by 540 fenestrations and 147 directional branches. Technical success was $94 \%$. Thirty-day mortality was $4.3 \%$, including a mortality of $1.8 \%$ for extent IV and $8.2 \%$ for extent I to III thoracoabdominal aortic aneurysms $(P=.03)$. Mortality decreased in the second half of clinical experience from $7.5 \%$ to $1.2 \%$, including a decrease of $3.3 \%$ to $0 \%$ for extent IV thoracoabdominal aortic aneurysms $(P=.12)$ and $15.6 \%$ to $2.4 \%$ for extent I to III thoracoabdominal aortic aneurysms $(P=.04)$. Early major adverse events occurred in 36 patients $(32 \%)$ with extent IV thoracoabdominal aortic aneurysms and 26 patients $(36 \%)$ with extent I to III thoracoabdominal aortic aneurysms, including spinal cord injury in 2 patients $(1.8 \%)$ and 4 patients $(3.2 \%)$, respectively. Mean follow-up was $21 \pm 20$ months. At 5 years, patient survival $(56 \%$ and $59 \%$, $P=.37)$ and freedom from any reintervention $(50 \%$ and $53 \%, P=.26)$ were similar in those with extent IV and extent I to III thoracoabdominal aortic aneurysms. Primary patency was $93 \%$ at 5 years.

Conclusions: Endovascular repair of thoracoabdominal aortic aneurysms can be performed with high technical success and low mortality and morbidity. However, the need for secondary reinterventions and continued graft surveillance represents major limitations compared with results of conventional open surgical repair. Long-term follow-up is needed before the widespread use of these techniques in younger or lower-risk patients. (J Thorac Cardiovasc Surg 2017;153:S32-41)

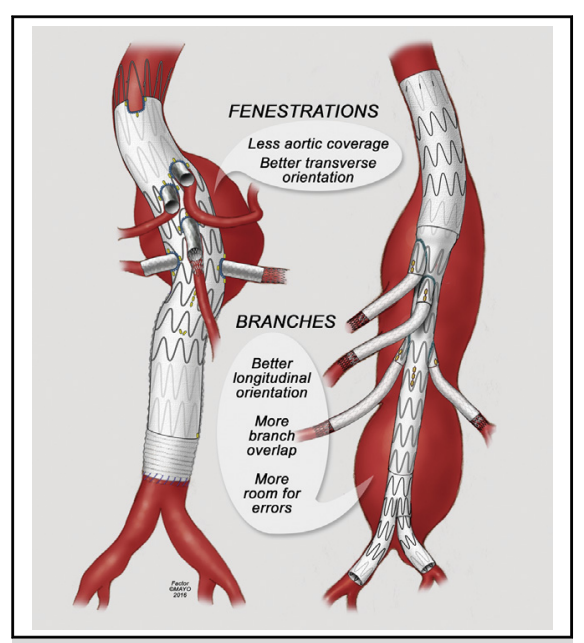

Branch incorporation can be done with reinforced fenestrations or branches.

Central Message

This article reviews the outcomes of novel fenestrated and branched endografts to treat TAAAs in a single center.

\section{Perspective}

Fenestrated and branched endografts have been used to treat TAAAs in elderly and higher-risk patients. This study shows low mortality (4\%), SCI $(3 \%)$, and dialysis rate $(1 \%)$, which compares favorably to historical results of conventional open repair in large centers. Rate of reinterventions was high because of endoleak of branch vessel stenosis.

See Editorial Commentary page S42.
From the advanced Endovascular Aortic Research Program, Division of Vascular and Endovascular Surgery; ${ }^{b}$ Department of Epidemiology and Biostatistics, Mayo Clinic, Rochester, Minn; and ${ }^{\mathrm{c} D i v i s i o n}$ of Vascular and Endovascular Surgery, Department of Surgery and Anatomy, University of São Paulo, Faculty of Medicine of Ribeirão Preto, Ribeirão Preto, Brazil.

L.R.dS. is sponsored by the Masters Degree Program from the Department of Surgery, Universidade Federal do Rio Grande do Sul.

Read at the 2016 Annual Aortic Symposium of the American Association of Thoracic Surgeons, New York, New York, May 12-13, 2016.
Received for publication May 31, 2016; revisions received Aug 24, 2016; accepted for publication Oct 6, 2016; available ahead of print Nov 17, 2016.

Address for reprints: Gustavo S. Oderich, MD, Gonda Vascular Center, Mayo Clinic, 200 First St SW, Rochester MN 55905 (E-mail: oderich.gustavo@mayo.edu). $0022-5223 / \$ 36.00$

Copyright (C) 2016 by The American Association for Thoracic Surgery http://dx.doi.org/10.1016/j.jtcvs.2016.10.008 


\section{Abbreviations and Acronyms \\ $\mathrm{F}-\mathrm{BEVAR}=$ fenestrated and branched endovascular aortic repair \\ IDE = investigational device exemption \\ MAE $=$ major adverse event \\ PMEG = physician-modified endovascular graft \\ SCI = spinal cord injury \\ TAAA $=$ thoracoabdominal aortic aneurysm \\ TASP $=$ temporary aneurysm sac perfusion}

\section{Scanning this QR code will take}

you to supplemental figures, tables,

and video for this article.

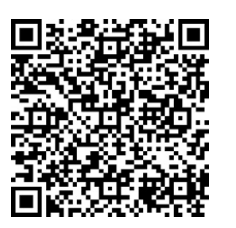

Fenestrated and branched stent-grafts have been increasingly used to extend the indications of endovascular aortic aneurysm repair to patients with complex aortic aneurysms involving the supra-aortic trunks, renal-mesenteric arteries, and iliac bifurcation. Since the first report of an endovascular repair of a thoracoabdominal aortic aneurysm (TAAA) by Chuter and colleagues ${ }^{1}$ in 2001, the technique has evolved from physician-modified endovascular grafts (PMEGs) to manufactured off-the-shelf or patient-specific endografts. ${ }^{1}$ Several refinements in device design, including reinforced fenestrations; straight, helical, internal, and external branches; preloaded catheters and guidewire systems; and lower profile fabric, have facilitated or simplified these procedures, accounting for the high technical success $(>95 \%)$ in most reports. ${ }^{2-6}$ The mortality rate has varied in the literature depending on clinical experience, learning curve, and adoption of specific protocols to reduce spinal cord injury (SCI), including staging, early limb reperfusion, and neuromonitoring. ${ }^{5-9}$ It is evident from these reports that development of dedicated teams and improvements in preoperative planning, patient selection, techniques of implantation, and perioperative intensive care have lowered perioperative mortality and paraplegia. The purpose of this study was to review the outcomes of endovascular repair using fenestrated and branched endovascular aortic repair (F-BEVAR) in consecutive patients treated at a single center.

\section{MATERIALS AND METHODS}

The study was approved by the Institutional Review Board of the Mayo Clinic. All patients consented for participation in clinical research studies. We identified all consecutive patients treated for TAAAs by F-BEVAR. All patients included in the study had at least more than 2 sealing stents above the celiac axis. Selection of fenestrated and branched endograft design evolved from PMEGs (2007-2013) to manufactured devices (2012-
2016), which were performed using Cook Zenith (Cook Medical Inc, Bloomington, Ind) off-the-shelf or patient-specific thoracoabdominal stentgrafts. Patients treated since 2013 were enrolled in a prospective physician-sponsored investigational device exemption (IDE) protocol (www.ClinicalTrials.gov, NCT 1937949 and 2089607).

The clinical data were retrospectively reviewed for demographics, clinical characteristics, cardiovascular risk factors, comorbidity scores, radiologic imaging, operative data, and outcomes. The early postoperative period was defined as within the first 30 days or within the hospital stay if more than 30 days. Follow-up consisted of clinical examination and diagnostic imaging before discharge and at 2, 6, and 12 months and annually thereafter. Imaging evaluation included computed tomography angiography or computed tomography without contrast and duplex ultrasound of the renal-mesenteric arteries. All imaging studies were independently evaluated by a group of vascular radiologists. Clinical and imaging follow-up was obtained prospectively in all patients treated under the IDE protocols. Among patients included in the prospective arm, adverse events were reviewed and adjudicated by an independent clinical event committee and Data Safety Monitoring Board.

\section{Statistical Analysis}

Data were managed and stored in a MEDIDATA Rave (New York, NY) database and analyzed using SAS 9.1 software (SAS Institute, Inc, Cary, $\mathrm{NC}$ ). Outcomes were analyzed separately for extent IV and extent I to III TAAAs using Crawford and colleagues' classification. ${ }^{10}$ The reporting standards of the Society for Vascular Surgery for endovascular repair of thoracic aneurysms were used to define technical success, aneurysm sac changes, endoleak, migration, and device integrity. ${ }^{11}$ Major adverse events (MAEs) were defined using a composite end point, which included any cause mortality, severe acute kidney injury ( $>50 \%$ decrease in estimated glomerular filtration rate), new-onset dialysis, myocardial infarction, respiratory failure requiring prolonged mechanical ventilation or reintubation, paraplegia (nonambulatory, grade 3a-3c Spinal Cord Injury Scale), ${ }^{11}$ stroke, bowel ischemia requiring surgical resection or intensification of medical therapy, and estimated blood loss more than 1 liter. Primary and secondary patency were defined by uninterrupted patency from index procedure until occlusion or any stent reintervention for stenosis and by an occlusion treated by surgical bypass or not suitable to endovascular salvage, respectively. Time-dependent outcomes were reported using Kaplan-Meier estimates. Differences were determined by the log-rank test. Results were reported as percent for categoric variables and mean \pm standard deviation for continuous variables. The Pearson's chi-square or Fisher exact test was used for analysis of categoric variables. Differences between means were tested with the 2-sided Student $t$ test, Wilcoxon rank-sum test, or Mann-Whitney test. Data are presented as median values or mean \pm standard deviation, as appropriate.

\section{Device Design}

Aneurysm morphology was determined by high-resolution computed tomography angiography datasets. Device design was planned using the centerline of flow measurements with a minimum proximal landing zone of $20 \mathrm{~mm}$ based on healthy aortic segments with a parallel aortic wall with no thrombus or calcium. The technique of device design, modification, and implantation of PMEGs has been described in detail ${ }^{12-14}$ and is summarized in the Video 1. Physician-modified fenestrated stent-grafts were customized onsite under a strict sterile technique using the Cook Zenith or TX2 platform (Cook Medical Inc) with 1 to 5 reinforced fenestrations or directional branches. A diameter-reducing wire was used to facilitate target vessel catheterization. For manufactured devices, the design consisted of an off-the-shelf multibranch stent-graft (t-Branch, Cook Medical Inc, Brisbane, Australia) or a patient-specific design with any combination of 1 to 5 fenestrations or branches (Figure 1). In general, directional branches were preferentially selected for longitudinally oriented vessels that originated from a large luminal aortic diameter 


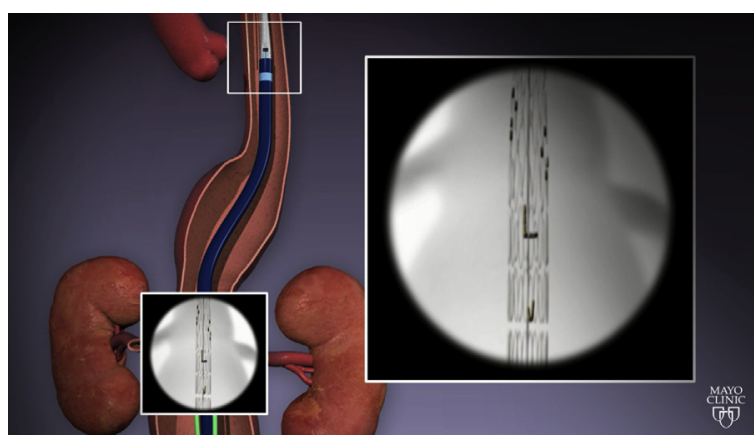

VIDEO 1. The technique of endovascular repair of a TAAA using fenestrated-branched stent-grafts is shown. Preoperative imaging is reviewed using centerline of flow analysis for measurements of lengths, angle, and clock position of the mesenteric and renal target vessels. A patientspecific stent-graft is designed with 2 directional branches for the celiac and superior mesenteric arteries and 2 renal fenestrations. The procedure is performed using transfemoral and brachial access. Once the target vessels are located, the device is oriented and introduced via the femoral approach. Preloaded catheters allow guidewires to be snared via the brachial approach. The device is partially deployed to the level of the mesenteric arteries. By using the brachial approach, the celiac and superior mesenteric arteries are accessed and the hydrophilic sheaths are advanced over stiff guidewires. The rest of the device is deployed below the renal arteries. The renal fenestrations and renal arteries are accessed via the femoral approach. The device is completely deployed, followed by sequential stenting of the renal and mesenteric arteries using covered stent-grafts. The repair is extended distally using a bifurcated stent-graft and iliac limbs. A completion angiography is performed to document patency of the side branches and absence of endoleak. Video available at: http://www.jtcvsonline.org/article/S00225223(16)31379-4/addons.

$(>30 \mathrm{~mm}$ ) (Figure E1), whereas fenestrations were selected for transversely originating vessels with an origin in narrow aortic segments (Figure E2).

\section{Alignment Stents}

Alignment stents were used in all vessels accommodated by fenestrations or directional branches. For fenestrations, iCAST balloonexpandable covered stents (Atrium Maquet, Hudson, $\mathrm{NH}$ ) were used with or without selective placement of self-expandable bare metal stents at the distal edge to prevent kinks. Directional branches were bridged to the target vessels using a Viabahn stent-graft (WL Gore \& Associates, Flagstaff, Ariz) for the renal arteries or a Fluency stent-graft (Bard, Covington, $\mathrm{Ga}$ ) for the celiac axis and superior mesenteric artery.

\section{Prevention of Spinal Cord Injury}

Our protocol of SCI prevention has been reported. ${ }^{9}$ Staged endovascular repair was indicated for extent I to II TAAAs (Figure E3). Subclavian and hypogastric artery revascularization was performed if needed to extend a landing zone. Perioperative measures included discontinuation of calcium channel blockers and angiotensin inhibitors 1 week before and up to 4 to 6 weeks after the procedure. Permissive hypertension with mean arterial pressure targeted at $80 \mathrm{~mm} \mathrm{Hg}$ or more was used in the first 72 hours after the operation. Transfusion of blood products was indicated in the first 48 hours after the procedure to keep a target hemoglobin $10 \mathrm{mg} / \mathrm{dL}$ or more and normal coagulation profile. Cerebrospinal fluid drainage was indicated routinely in all extent I to III TAAAs and has been used for extent IV TAAAs since 2013. Total intravenous anesthesia was used to allow

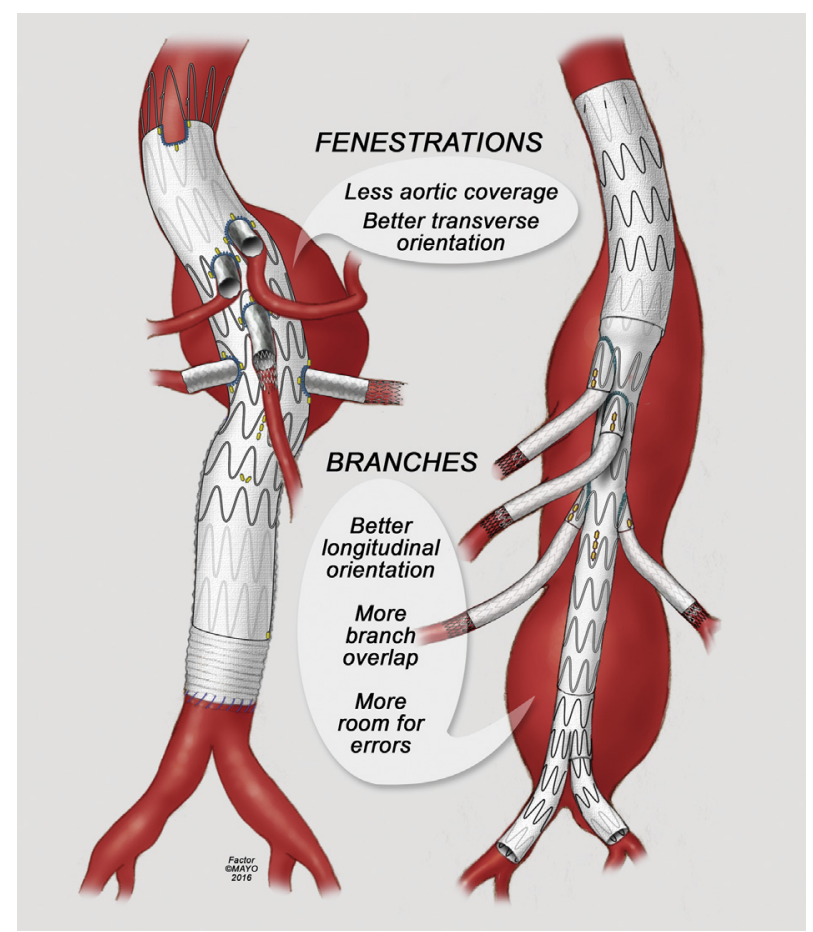

FIGURE 1. Advantages of patient-specific fenestrated and off-the-shelf multibranched stent-grafts.

intraoperative neurophysiologic monitoring of motor-evoked potential and somatosensory-evoked potentials. A $75 \%$ or greater reduction from baseline evoked potential amplitude was considered to be significant and triggered standardized maneuvers (Figure E4) to increase spinal cord perfusion and restore flow to the pelvis and lower extremities.

\section{RESULTS \\ Study Patients}

There were 185 patients, 134 male (72\%) and 51 female $(28 \%)$, with a mean age of $75 \pm 7$ years (Table 1). Aneurysm classification was extent IV TAAA in 112 patients $(60 \%)$ and extent I to III TAAA in 73 patients $(40 \%)$. The maximum aneurysm diameter averaged $66 \pm 13 \mathrm{~mm}$. Patients in both groups had similar demographics and cardiovascular risk factors. Society for Vascular Surgery comorbidity scores were higher in patients with extent IV TAAAs ( $13 \pm 4$ vs $12 \pm 4, P=.029$ ), but patients with extent I to III TAAAs had more prior aortic repairs $(54 \%$ vs $27 \%, P<.001)$ and abdominal operations $(57 \%$ vs $41 \%, P=.035)$. For the entire cohort, the most prevalent risk factors were hypertension in 164 patients $(90 \%)$, cigarette smoking in 159 patients (87\%), hyperlipidemia in 149 patients $(81 \%)$, ischemic cardiomyopathy in 112 patients $(61 \%)$, chronic obstructive pulmonary disease in 88 patients $(48 \%)$, and stage III to $\mathrm{V}$ chronic kidney disease in 60 patients $(32 \%)$.

\section{Stent-Graft Design}

Endovascular repair was performed using PMEGs in 78 patients $(42 \%, 44$ extent IV and 37 extent I-III) 
TABLE 1. Demographics and clinical characteristics of 185 patients treated for thoracoabdominal aortic aneurysms with fenestrated-branched stent-grafts

\begin{tabular}{|c|c|c|c|c|c|c|c|c|c|c|}
\hline \multirow[b]{2}{*}{ Variable } & \multicolumn{3}{|c|}{ Extent I-III } & \multicolumn{3}{|c|}{ Extent IV } & \multirow[b]{2}{*}{$\begin{array}{c}P \\
\text { value } \\
\end{array}$} & \multicolumn{3}{|c|}{ Overall } \\
\hline & $(\mathbf{N}=73)$ & $\begin{array}{l} \pm \text { SD } \\
\text { or } \%\end{array}$ & $\begin{array}{l}\text { Median } \\
(\mathrm{Q1}, \mathrm{Q3})\end{array}$ & $(N=112)$ & $\begin{array}{l} \pm \text { SD } \\
\text { or } \%\end{array}$ & $\begin{array}{l}\text { Median } \\
(\mathrm{Q1}, \mathrm{Q3})\end{array}$ & & $(\mathbf{N}=\mathbf{1 8 5})$ & $\begin{array}{l} \pm \text { SD } \\
\text { or } \%\end{array}$ & $\begin{array}{l}\text { Median } \\
(Q 1, Q 3)\end{array}$ \\
\hline Age (y) & 74 & \pm 7 & $74(69,79)$ & 75 & \pm 7 & $76(72.5,79.5)$ & $.12 *$ & 75 & \pm 7 & $75(70,79)$ \\
\hline Male gender & 48 & 66 & & 86 & 77 & & .1 & 134 & 72 & \\
\hline \multicolumn{11}{|l|}{ Cardiovascular risk factors } \\
\hline Hypertension & 67 & 94 & & 97 & 87 & & .09 & 164 & 90 & \\
\hline Smoker & 63 & 89 & & 96 & 86 & & .56 & 159 & 87 & \\
\hline Hypercholesterolemia & 59 & 83 & & 90 & 80 & & .64 & 149 & 81 & \\
\hline Coronary artery disease & 43 & 61 & & 69 & 62 & & .89 & 112 & 61 & \\
\hline $\begin{array}{l}\text { Chronic obstructive } \\
\text { pulmonary disease }\end{array}$ & 37 & 52 & & 51 & 46 & & .39 & 88 & 48 & \\
\hline MI & 23 & 32 & & 45 & 40 & & .29 & 68 & 37 & \\
\hline Arrhythmia & 9 & 13 & & 23 & 21 & & .17 & 32 & 17 & \\
\hline Diabetes mellitus & 10 & 14 & & 21 & 19 & & .41 & 31 & 17 & \\
\hline $\mathrm{CHF}$ & 7 & 10 & & 19 & 17 & & .18 & 26 & 14 & \\
\hline Stroke/TIA & 7 & 10 & & 13 & 12 & & .71 & 20 & 11 & \\
\hline \multicolumn{11}{|c|}{ Preoperative evaluation and comorbidity scores } \\
\hline Positive cardiac stress test & 9 & 14 & & 28 & 29 & & .035 & 37 & 23 & \\
\hline Baseline creatinine (mg/dL) & $1.4 \dagger$ & \pm 1.4 & $1.0(0.9,1.3)$ & 1.3 & \pm 0.5 & $1.1(1.0,1.4)$ & $.32 *$ & 1.3 & \pm 1 & $1.1(0.9,1.4)$ \\
\hline $\begin{array}{l}\text { Baseline glomerular filtration } \\
\text { rate }\left(\mathrm{mL} / \mathrm{min} / 1.73 \mathrm{~m}^{2}\right)\end{array}$ & $62 \dagger$ & \pm 22 & $65(48.5,75)$ & 60 & \pm 20 & $58.5(45,72)$ & $.54^{*}$ & 60 & \pm 21 & $60.5(45.5,73)$ \\
\hline $\begin{array}{l}\text { Chronic kidney disease stage } \\
\text { III-V }\end{array}$ & 23 & 32 & & 37 & 33 & & .83 & 60 & 32 & \\
\hline Stage III & 19 & 83 & & 30 & 81 & & & 49 & 82 & \\
\hline Stage IV & 2 & 9 & & 6 & 16 & & & 8 & 13 & \\
\hline Stage V & 2 & 9 & & 1 & 3 & & & 3 & 5 & \\
\hline BMI $\left(\mathrm{kg} / \mathrm{m}^{2}\right)$ & 27 & \pm 5 & & 29 & \pm 6 & & .036 & 28 & \pm 6 & \\
\hline ASA clinical score & & & & & & & .13 & & & \\
\hline I & 4 & 6 & & 12 & 11 & & & 16 & 9 & \\
\hline II & 34 & 47 & & 57 & 51 & & & 91 & 49 & \\
\hline III & 27 & 38 & & 34 & 30 & & & 61 & 33 & \\
\hline IV & 4 & 6 & & 9 & 8 & & & 13 & 7 & \\
\hline $\mathrm{V}$ & 3 & 4 & & 0 & 0 & & & 3 & 2 & \\
\hline SVS total score $(0-30)$ & 12 & \pm 4 & & 13 & \pm 4 & & .029 & 13 & \pm 4 & \\
\hline Connective tissue disorder & 2 & 3 & & 2 & 2 & & .91 & 4 & 2 & \\
\hline Peripheral arterial disease & 22 & 31 & & 38 & 34 & & .68 & 60 & 33 & \\
\hline \multicolumn{11}{|l|}{ Medical history } \\
\hline Previous aortic surgery & 39 & 54 & & 30 & 27 & & $<.001$ & 69 & 38 & \\
\hline Previous abdominal surgery & 41 & 57 & & 46 & 41 & & .035 & 87 & 47 & \\
\hline $\begin{array}{l}\text { Endovascular aortic } \\
\text { intervention }\end{array}$ & 18 & 25 & & 17 & 15 & & .1 & 35 & 19 & \\
\hline History of malignancy & 16 & 23 & & 27 & 24 & & .81 & 43 & 23 & \\
\hline $\begin{array}{l}\text { Family history of aortic } \\
\text { aneurysm }\end{array}$ & 10 & 15 & & 10 & 12 & & .57 & 20 & 13 & \\
\hline
\end{tabular}

Continuous data are presented as mean \pm SD; categoric data are given in percentage. Q1 and Q3 refer to 25th and 75th percentiles, respectively. $N$, Number of patients; $S D$, standard deviation; $M I$, myocardial infarction; $C H F$, congestive heart failure; TIA, transient ischemic attack; BMI, body mass index; $A S A$, American Society of Anesthesiologists; $S V S$, Society for Vascular Surgery. *Equal variance $t$ test. †One missing entry.

and manufactured devices in 107 patients $(58 \%, 68$ extent IV and 36 extent I-III). A total of 681 renalmesenteric arteries (170 celiac, 183 superior mesenteric, 161 right renal, and 167 left renal arteries) were incorporated by 538 fenestrations and 143 directional branches, with a mean of 3.7 vessels per patient (Table E1).
Among patients treated for extent IV TAAAs, there were 421 vessels incorporated by 393 fenestrations $(93 \%)$ and 28 directional branches $(7 \%)$. For extent I to III TAAAs, there were 260 vessels incorporated by 145 fenestrations $(56 \%)$ and 115 directional branches (44\%). 


\section{Endovascular Repair}

All procedures were performed in a hybrid endovascular room with a fixed imaging unit using general endotracheal anesthesia in 184 patients (99\%) (Table 2). Cerebrospinal fluid drainage was used in 147 patients $(79 \%)$, and neuromonitoring was used in 110 patients $(59 \%)$. Ninety-six patients $(52 \%)$ had total percutaneous transfemoral approach with a preclosure technique, and 19 patients required an iliac conduit $(10 \%)$. Total volume of contrast and fluoroscopy time averaged $183 \pm 72 \mathrm{~mL}$ and $95 \pm 44$ minutes, respectively. Contrast use and fluoroscopy time were significantly higher in patients with extent I to III TAAAs $(P<.001)$. Technical success, defined by placement of the aortic stent and all intended side branches, was achieved in 174 patients (94\%) and was $96 \%$ for extent IV TAAAs $(96 \%)$ and $90 \%$ for extent I to III TAAAs $(P=.09)$. Estimated blood loss was $903 \pm 1164 \mathrm{~mL}$ with no difference between groups. Eighty-one patients $(44 \%)$ received transfusion of packed red blood cells during the procedure.

\section{Mortality}

There were 8 deaths $(4.3 \%)$ within 30 days and 7 deaths $(3.7 \%)$ within the hospital stay (Tables 3 and E2). There were 2 deaths among patients treated for extent IV TAAA repair $(1.8 \%)$ and 6 deaths in those treated for extent I to III TAAA repair $(8.2 \%, P=.035)$ in the first 30 days. All deaths occurred among patients treated by PMEGs, and there was no mortality in patients treated using manufactured devices under the prospective IDE study. Time to accumulate the first half of the experience was 78 months, compared with 21 months for the second half of the experience (Figure 2). During this period, mortality decreased from $7.5 \%$ to $1.2 \%(P=.03)$, including a decrease of $3.3 \%$ to $0 \%$ for extent IV TAAAs $(P=.19)$ and $15.6 \%$ to $2.4 \%$ for extent I to III TAAAs $(P=.04)$. Causes of death were ischemic colitis with multisystem organ failure in 2 patients and myocardial infarction, cardiac arrest after rapid ventricular pacing, pulmonary aspiration, stroke, acute renal failure, and unknown cause in 1 patient each.

\section{Major Adverse Events}

There were 107 MAEs in 67 patients $(36 \%)$ in the entire cohort (Table 3). In the early follow-up ( $<30$ days), 62 patients had MAEs, including 36 patients $(32 \%)$ treated for extent IV TAAAs and 26 patients $(36 \%)$ treated for extent I to III TAAAs $(P=.91)$. The most common MAE in the first 30 days was estimated blood loss of more than 1 liter in 41 patients $(22 \%)$, followed by acute kidney injury in 23 patients $(12 \%)$, respiratory failure requiring reintubation in 10 patients $(5 \%)$, myocardial infarction in 9 patients $(5 \%)$, paraplegia (grade 3a-c SCI) in 6 patients $(4.3 \%)$, stroke in 5 patients $(3 \%)$, and bowel ischemia requiring surgical resection or intensification of therapy in 4 patients $(2 \%)$. Nine patients $(4.8 \%)$ had spinal cord injuries, including 3 treated for extent IV TAAAs $(2.7 \%)$ and 6 treated for extent I to III TAAAs $(8.2 \%)$. Of these, 3 patients had grade I to II SCIs and were ambulatory. Six patients $(3.6 \%)$ had grade IIIa to IIIc nonambulatory injuries, including 2 with extent IV TAAAs $(1.8 \%)$ and 4 with extent I to III TAAAs

TABLE 2. Procedural details in 185 patients treated for thoracoabdominal aortic aneurysms with fenestrated-branched stent-grafts

\begin{tabular}{|c|c|c|c|c|c|c|c|}
\hline \multirow{3}{*}{$\begin{array}{l}\text { Variable } \\
\text { General anesthesia }\end{array}$} & \multirow{2}{*}{\multicolumn{2}{|c|}{$\begin{array}{c}\text { Extent I-III } \\
(\mathrm{N}=73)\end{array}$}} & \multirow{2}{*}{\multicolumn{2}{|c|}{$\begin{array}{l}\text { Extent IV } \\
(\mathrm{N}=112)\end{array}$}} & \multirow{3}{*}{$P$ value } & \multirow{2}{*}{\multicolumn{2}{|c|}{$\begin{array}{c}\text { Overall } \\
(\mathrm{N}=\mathbf{1 8 5})\end{array}$}} \\
\hline & & & & & & & \\
\hline & 72 & $99 \%$ & 112 & $100 \%$ & & 184 & $99 \%$ \\
\hline Cerebrospinal fluid drainage & 72 & $99 \%$ & 75 & $67 \%$ & $<.001$ & 147 & $79 \%$ \\
\hline $\mathrm{SSP} /$ motor-evoked potentials & 50 & $68 \%$ & 60 & $54 \%$ & .043 & 110 & $59 \%$ \\
\hline Percutaneous & 37 & $51 \%$ & 59 & $54 \%$ & .7 & 96 & $52 \%$ \\
\hline Amount of contrast used (mL) & 206 & \pm 84 & 168 & \pm 58 & $<.001$ & 183 & \pm 72 \\
\hline Total fluoroscopy time (min) & 105 & \pm 54 & 89 & \pm 35 & .015 & 95 & \pm 44 \\
\hline Estimated blood loss (mL) & 1080 & \pm 1427 & 787 & \pm 945 & .09 & 903 & \pm 1164 \\
\hline \multicolumn{8}{|l|}{ Transfusion of blood products } \\
\hline PRBC & 42 & $58 \%$ & 39 & $35 \%$ & .002 & 81 & $44 \%$ \\
\hline Volume (mL) & 1094 & \pm 966 & 899 & \pm 883 & .3 & 1000 & \pm 926 \\
\hline FFP & 14 & $19 \%$ & 11 & $10 \%$ & .07 & 25 & $14 \%$ \\
\hline Volume (mL) & 847 & \pm 354 & 773 & \pm 711 & .7 & 814 & \pm 529 \\
\hline Platelets & 16 & $22 \%$ & 13 & $12 \%$ & .06 & 29 & $16 \%$ \\
\hline Volume (mL) & 426 & \pm 170 & 587 & \pm 337 & .1 & 498 & \pm 266 \\
\hline Cryoprecipitate & 8 & $11 \%$ & 3 & $3 \%$ & .021 & 11 & $6 \%$ \\
\hline Volume (mL) & 187 & \pm 36 & 240 & \pm 42 & .1 & 202 & \pm 43 \\
\hline Hypogastric branches (unilateral or bilateral) & 1 & $1.4 \%$ & 4 & $3.6 \%$ & .58 & 5 & $2.7 \%$ \\
\hline No. of vessels stented per patient (mean) & 3.6 & \pm 0.84 & 4 & \pm 0.72 & .05 & 3.7 & \pm 0.77 \\
\hline Iliac conduit & 5 & $6.8 \%$ & 11 & $9.8 \%$ & .87 & 16 & $8.6 \%$ \\
\hline
\end{tabular}

$N$, Number of patients; SSP, somatosensory evoked potential; $P R B C$, packed red blood cells; FFP, Fresh-frozen plasma. 
TABLE 3. Early mortality and major adverse events less than 30 days in 185 patients treated for thoracoabdominal aortic aneurysms with fenestrated-branched endovascular repair

\begin{tabular}{|c|c|c|c|c|c|c|c|}
\hline \multirow{3}{*}{$\begin{array}{l}\text { Variable } \\
\text { Any cause of mortality }\end{array}$} & \multirow{2}{*}{\multicolumn{2}{|c|}{$\begin{array}{l}\text { Type I-III } \\
(\mathrm{N}=73) \\
\end{array}$}} & \multirow{2}{*}{\multicolumn{2}{|c|}{$\begin{array}{c}\text { Type IV } \\
(\mathrm{N}=112)\end{array}$}} & \multirow{3}{*}{$\frac{P \text { value }}{.83}$} & \multirow{2}{*}{\multicolumn{2}{|c|}{$\begin{array}{c}\text { Overall } \\
(\mathbf{N}=\mathbf{1 8 5})\end{array}$}} \\
\hline & & & & & & & \\
\hline & 6 & $8 \%$ & 2 & $2 \%$ & & 8 & $4 \%$ \\
\hline Any MAE & 26 & $36 \%$ & 36 & $32 \%$ & .62 & 62 & $34 \%$ \\
\hline Estimate blood loss $>1000 \mathrm{~mL}$ & 20 & $27 \%$ & 21 & $19 \%$ & .17 & 41 & $22 \%$ \\
\hline Acute kidney injury ( $>50 \%$ decrease in GFR) & 7 & $11 \%$ & 14 & $13 \%$ & .62 & 21 & $11 \%$ \\
\hline New-onset dialysis* & 1 & $1.3 \%$ & 1 & $0.8 \%$ & .74 & 2 & $1 \%$ \\
\hline MI & 1 & $1 \%$ & 8 & $7 \%$ & .07 & 9 & $5 \%$ \\
\hline Respiratory failure & 7 & $10 \%$ & 3 & $3 \%$ & .042 & 10 & $5 \%$ \\
\hline Paraplegia (SCI grade 3a-3c) & 4 & $5 \%$ & 2 & $2 \%$ & .16 & 6 & $3 \%$ \\
\hline Stroke & 2 & $3 \%$ & 3 & $3 \%$ & .98 & 5 & $3 \%$ \\
\hline $\begin{array}{l}\text { Bowel ischemia requiring intensification } \\
\text { of medical therapy }\end{array}$ & 1 & $1 \%$ & 3 & $3 \%$ & .55 & 4 & $2 \%$ \\
\hline
\end{tabular}

$N$, Number of patients; $M A E$, major adverse event; $G F R$, glomerular filtration rate; $M I$, myocardial infarction; $S C I$, spinal cord injury. $*$ One patient discontinued dialysis after 12 days.

$(5.5 \%)$. Four SCIs were immediate, and 5 SCIs were delayed. For the entire cohort, the rate of SCI decreased in the second half experience from $5.4 \%$ to $1.1 \%$ $(P=.09)$. Two patients $(1 \%)$ required new-onset dialysis, which was discontinued in 1 patient after 12 days and in the other patient after 4 months.

\section{Late Outcomes}

Mean follow-up averaged $22 \pm 20$ months (range, 1-97). Follow-up imaging studies were obtained in 179 patients with a mean of 3 studies per patient. Follow-up was complete in all 105 patients treated with manufactured devices under a prospective IDE study. Patient survival at 5 years was similar for extent IV and extent I to III TAAAs (Figure 3), 56\% $\pm 10 \%$ and $59 \% \pm 9 \%(P=.37)$. There was 1 late aneurysm-related death due to rupture of the descending thoracic aortic dissection, which occurred proximal to a TAAA branched endograft used to treat an extent III TAAA. There were no conversions to open surgical repair or ruptures within the treated aortic segment.

Fifty-three patients $(29 \%)$ required 68 secondary reinterventions, which were performed within the first 30 days in

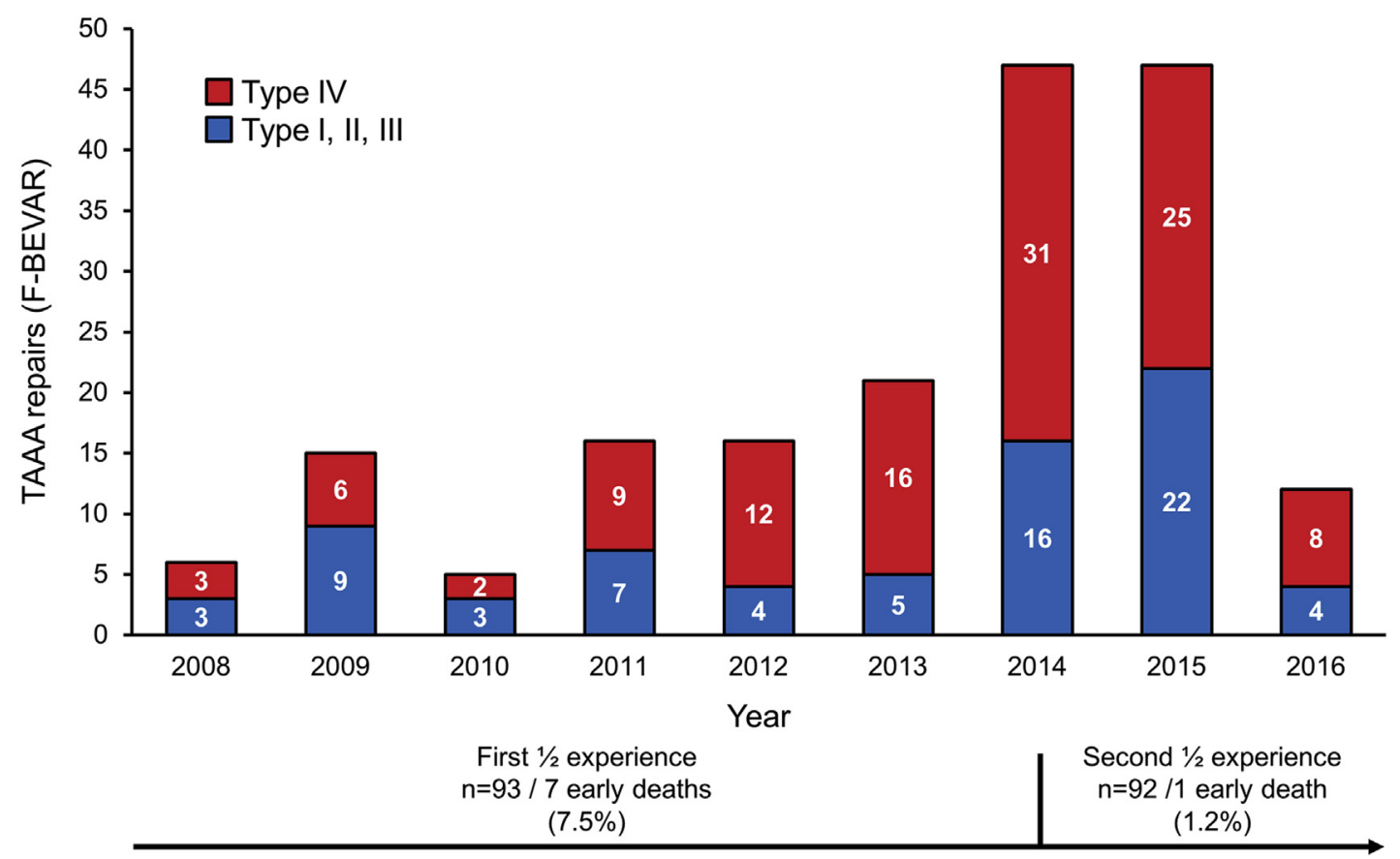

FIGURE 2. Graph of number of consecutive patients treated for type IV and type I to III TAAAs between 2008 and March 2016. Note the decrease in mortality from $7.5 \%$ in the first half to $1.2 \%$ in the second half experience $(P=.04)$. TAAA, Thoracoabdominal aortic aneurysm; $F-B E V A R$, fenestrated and branched endovascular aortic repair. 


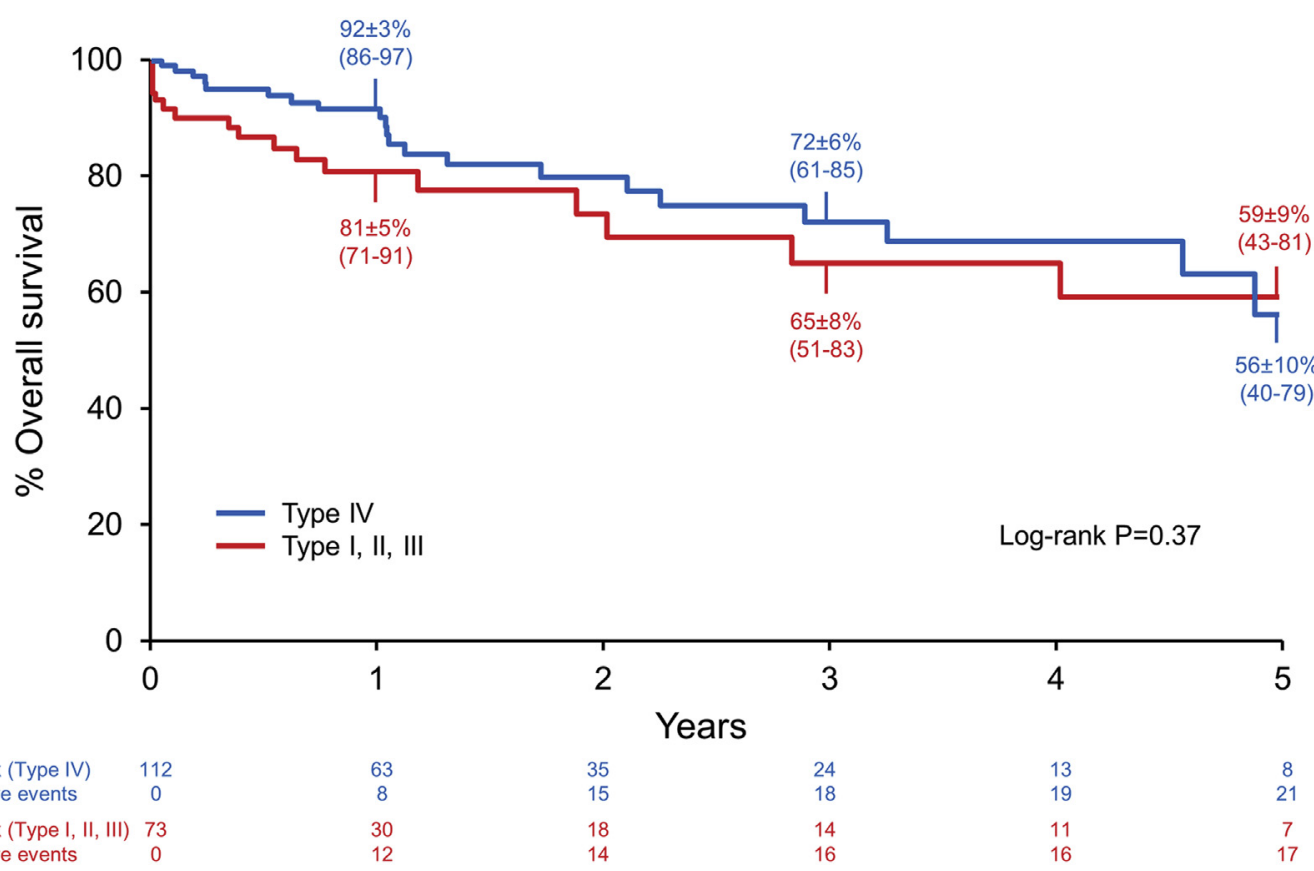

FIGURE 3. Kaplan-Meier estimates of overall patient survival in 185 consecutive patients treated for type IV and type I to III TAAAs between 2008 and March 2016.

26 patients $(14 \%, 31$ procedures) and after 30 days in 27 patients $(15 \%, 37$ procedures) (Tables 4 and E3). These included 48 aortic-related procedures (46 percutaneous, 2 open surgical) and 20 nonaortic-related procedures. The most common indications for a reintervention were endoleak in 23 procedures $(25 \% ; 17$ type III, 5 type II, 1 type I) or target vessel stenosis or kink in 13 procedures $(19 \%)$. Freedom from any reintervention was $50 \% \pm 9 \%$

TABLE 4. Description of early and late reinterventions in patients treated by fenestrated and branched endovascular repair for thoracoabdominal aortic aneurysms

\begin{tabular}{|c|c|c|c|c|c|c|}
\hline \multirow[b]{2}{*}{ Types of reinterventions } & \multicolumn{2}{|c|}{$<\mathbf{3 0}$ d } & \multicolumn{2}{|c|}{$>\mathbf{3 0} \mathbf{d}$} & \multicolumn{2}{|c|}{ Total } \\
\hline & $\mathbf{N}=\mathbf{1 8 5}$ & $\%$ & $\mathbf{N}=\mathbf{1 7 7}$ & $\%$ & $\mathbf{N}=\mathbf{1 8 5}$ & $\%$ \\
\hline \multicolumn{7}{|l|}{ Aortic } \\
\hline Device or branch stenosis/kinking/occlusion & 6 & 3 & 7 & 4 & 13 & 7 \\
\hline Device or branch migration & 1 & 1 & 0 & 0 & 1 & 1 \\
\hline Aneurysm degeneration & 0 & 0 & 2 & 1 & 2 & 1 \\
\hline \multicolumn{7}{|l|}{ Endoleak } \\
\hline Type I & 0 & 0 & 1 & 1 & 1 & 1 \\
\hline Type II & 0 & 0 & 1 & 1 & 1 & 1 \\
\hline Type III & 6 & 3 & 11 & 6 & 17 & 9 \\
\hline Combined & 0 & 0 & 4 & 2 & 4 & 2 \\
\hline Endotension (V) & 0 & 0 & 1 & 1 & 1 & 1 \\
\hline Type A dissection & 1 & 1 & 0 & 0 & 1 & 1 \\
\hline Type $\mathrm{B}$ dissection & 1 & 1 & 0 & 0 & 2 & 1 \\
\hline Planned reintervention (SCI prevention protocol) & 6 & 3 & 0 & 0 & 6 & 3 \\
\hline \multicolumn{7}{|l|}{ Type of reintervention } \\
\hline Percutaneous & 19 & 10 & 27 & 15 & 46 & 25 \\
\hline Surgical & 2 & 1 & 0 & 0 & 2 & 1 \\
\hline Total & 21 & 11 & 27 & 15 & 48 & 26 \\
\hline \multicolumn{7}{|l|}{ Nonaortic } \\
\hline Access site complications & 5 & 3 & 8 & 5 & 13 & 7 \\
\hline Laparotomy & 4 & 2 & 2 & 1 & 6 & 3 \\
\hline Lower-limb fasciotomy & 1 & 1 & 0 & 0 & 1 & 1 \\
\hline Total & 10 & 5 & 10 & 6 & 20 & 11 \\
\hline
\end{tabular}

$N$, Number of patients; $S C I$, spinal cord injury. 
for extent IV and $53 \% \pm 10 \%$ for extent I to III at 5 years $(P=.26)$ (Figure 4).

Eighty-five patients had at least 1 endoleak identified by follow-up computed tomography angiography, including type I endoleak in 2 patients (type Ic), type II endoleak in 55 patients, type III endoleak in 29 patients, and indeterminate endoleak in 12 patients. Of these, 23 patients had reinterventions, including $17(9 \%)$ who had treatment of type III endoleaks, in whom $12(6 \%)$ were due to the attachment of the fenestration and alignment stent.

Thirteen patients $(7 \%)$ had reinterventions to treat target vessel stenosis, kink, or occlusion. Primary target vessel patency at 1 and 5 years was $94 \% \pm 0.9 \%$ and $93 \% \pm 2 \%$, respectively (Figure E5).

\section{DISCUSSION}

Fenestrated and branched stent-grafts were introduced as an alternative to treat complex aneurysms in higher-risk patients who were not considered good candidates for open surgical repair. The indications of F-BEVAR have been broadened to patients with chronic dissection or failed aortic repair, and intermediate-risk patients. ${ }^{15,16}$ This report reflects the evolution of F-BEVAR techniques in our institution. Over nearly a decade of experience, the use of PMEGs was discontinued because of improved quality control, operating room efficiency, design versatility, and better reimbursement of manufactured devices, along with compliance with federal regulations and lack of long-term data for PMEGs. We also incorporated a number of adjunctive techniques, such as staging for extent I to II repairs and routine use of neuromonitoring, cerebrospinal fluid drainage, and early limb reperfusion. ${ }^{9}$ Overall, our results demonstrate low morbidity and mortality $(4.3 \%)$, which have declined significantly in the second half experience ( $7.5 \%$ to $1.2 \%)$. However, further technical refinements are needed to address the high rate of percutaneous aortic-related reinterventions to treat type III endoleaks or branch stenosis.

Stent design for TAAA repair continues to evolve with the primary goals of facilitating technical implantation and optimizing branch outcomes. Although there is controversy with respect to the ideal configuration, most experts agree that directional branches are easier to implant, have longer attachment to the aortic component, and rarely lead to endoleaks. ${ }^{2,17-19}$ These devices are well suited for down-going vessels that have an origin from the large aortic lumen. Conversely, fenestrations require precise planning and implantation, and are ideal for vessels that have an origin from the narrow aortic lumen and that are oriented in a transverse or up-going angle, such as the renal arteries in patients with extent IV and extent I TAAAs. ${ }^{19,20}$ Because of some reports of higher rates of renal occlusion for directional branches (up to $10 \%$ in 5 years), several experts have advocated the preferential use of fenestrations, which have low occlusion rates $(<2 \%) .{ }^{17-19}$ However, fenestrations may lead to type III endoleaks $(12 \%)$ due to branch disconnection or short overlap. ${ }^{3}$ Our rate of type III endoleak $(12 \%)$ from fenestrations was nearly identical to what has been reported by Eagleton and colleagues ${ }^{3}$ in a review of 354 patients treated for extent I to III TAAAs. To reduce rates of type III endoleaks, occlusions, and reinterventions, newer designs should focus on improvements in overlapping

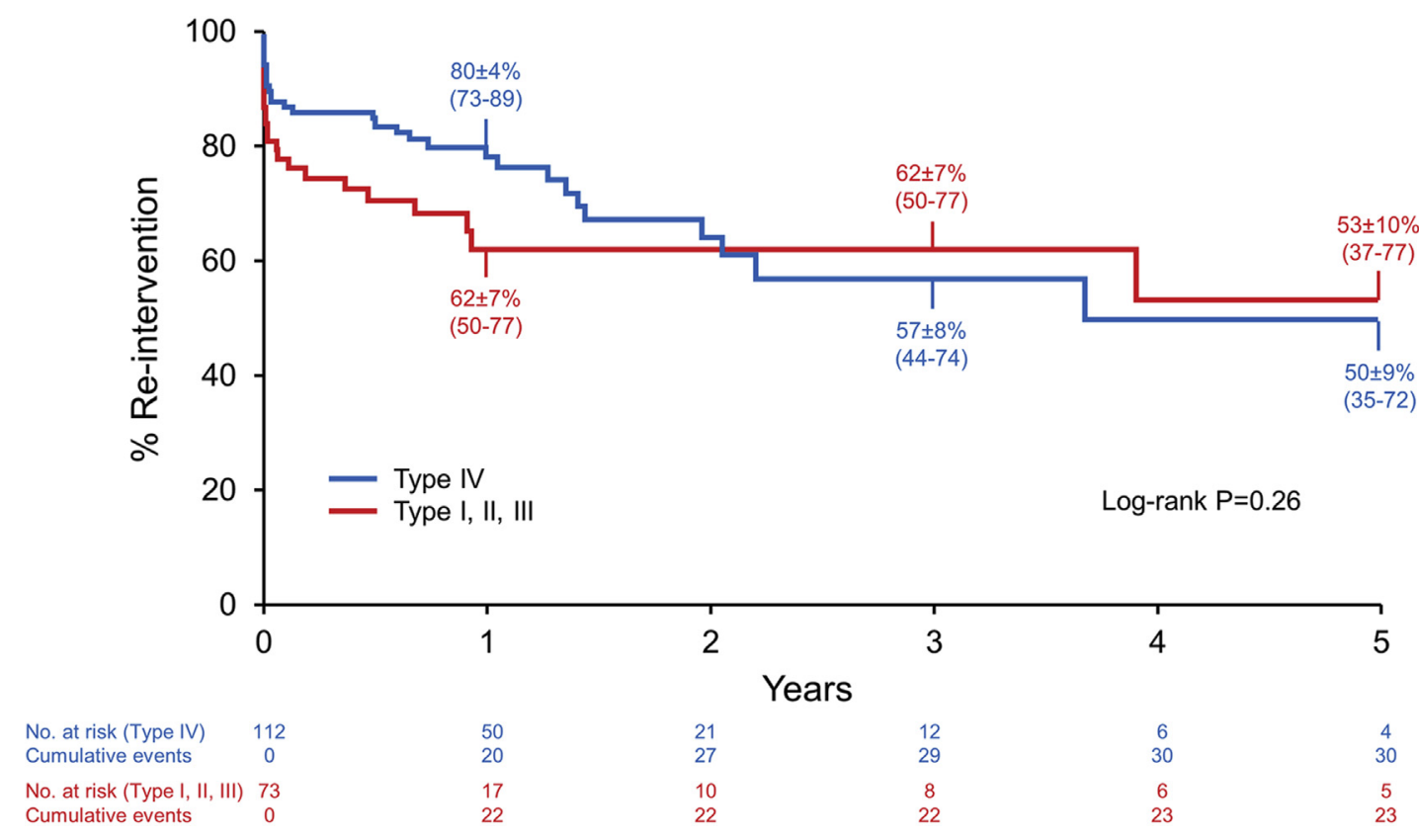

FIGURE 4. Kaplan-Meier estimates of freedom from any aortic and nonaortic-related reintervention in 185 consecutive patients treated for type IV and type I to III TAAAs between 2008 and March 2016. 
segments, bridging stent technology, and antiplatelet therapy.

The most feared complication of TAAA repair is paraplegia. Rates of SCI have varied from as low as $1.2 \%$ to as high as $31 \%{ }^{6-8}$ The risk is increased by extensive aortic coverage, occlusion of spinal collaterals, perioperative hypotension, lower-limb ischemia, and use of single-stage repair. Greenberg and colleagues ${ }^{21}$ reported a nonsignificant trend toward lower rates of SCI with endovascular repair $(4.3 \%)$ compared with open surgical repair (7.8\%) among 724 patients treated for TAAAs. For extent II TAAAs, both open (22\%) and endovascular (19\%) repair were associated with high rates of SCI. Subsequently, Eagleton and colleagues ${ }^{22}$ presented the results of 1251 patients with an overall rate of SCI of $2.9 \%$. The authors found that subclavian or hypogastric artery occlusion predicted the immediate onset of paraplegia and lack of recovery.

The concept of adaptation of collateral spinal network has been studied in experimental models, with some early experience in humans. ${ }^{23,24}$ Strategies for staged segmental arterial coverage using endovascular techniques include proximal thoracic endovascular aortic repair (Figure E1), temporary aneurysm sac perfusion (TASP), or intentional coil embolization of intercostal arteries. ${ }^{4,7}$ O'Callaghan and colleagues ${ }^{7}$ reported on 87 patients treated for extent II TAAAs using a single or 2-stage approach. In that study, staged repair was associated with no early mortality $(0 \%$ vs $19 \%$ ) or permanent paraplegia ( $0 \%$ vs $16 \%)$. TASP requires specially designed perfusion branches to induce an endoleak, leaving the repair incomplete. Kasprzak and colleagues ${ }^{4}$ reported a decrease in paraplegia risk from $21 \%$ to $2.5 \%$ using TASP. Although we have used perfusion branches in 6 patients with good outcomes, we no longer apply the technique because of potential risk of disseminated intravascular coagulopathy from high-flow endoleaks and the need to place all stents within a single-stage procedure, which prolongs operating time. Etz and colleagues ${ }^{25}$ recently reported on 2 patients who had intentional coil embolization of intercostal arteries before endovascular or open TAAA repair. Although this technique provides reliable segmental arterial occlusion, it adds a potential risk of embolization from catheter manipulations and requires that all stents be placed in a single operation. Maurel and associates ${ }^{8}$ reported lower rates of SCI ( $2 \%$ vs $25 \%$ ) using early limb reperfusion. Our improved rates of SCI reflect better patient selection and techniques of implantation, as well as routine use of the preventive measures outlined earlier.

Branch stability continues to be a focus of research. The ideal branch should optimize flow and aneurysm seal, avoiding endoleak, migration, fracture, kinks, stenosis, or occlusions. Mastracci and colleagues ${ }^{18}$ reported a 10 -year experience with 1679 target vessels using a composite end point to define branch stability, which included any endoleak, stenosis, occlusion, disconnection, or reintervention in a branch. Freedom from branch-related events was $89 \%$ in 5 years and $70 \%$ in 10 years. ${ }^{18}$ O'Callaghan and associates $^{26}$ reported that most type I endoleaks after fenestrated endografts became evident after 2 to 5 years, and that patients with type I endoleaks had shortened survival. Fortunately, with proper planning, the risk of type I endoleak is low, as evidenced by our study $(0.5 \%)$.

Endovascular aortic repair continues to be plagued by high reintervention rates, and it is logical that devices with multiple branches have more modules subjected to risk of failure. In our study, most reinterventions were done using percutaneous technique under local anesthesia, often as an outpatient. Coselli and colleagues ${ }^{27}$ recently reported the largest worldwide experience in a seminal study of 3309 patients treated for TAAA by open surgical repair over 2 decades. Thirty-day mortality was $7.5 \%$ for the entire cohort and $6.5 \%$ for elective cases, including a mortality of $5.1 \%$ for extent IV and $8 \%$ for extent I to III TAAAs. The mean age of patients was 66 years, and one third of the patients had aortic dissections. Although mortality rates were remarkably low compared with other reports, there were significant complications, including SCI $(9.6 \%)$, dialysis $(7.6 \%)$, and tracheostomy $(8.5 \%)$. The rate of any reintervention, which should factor all reoperations for bleeding, tracheostomy, wound problems, hernias, and other gastrointestinal complications, was not reported. However, freedom from aortic failure was remarkably low at $94 \%$ in 15 years, indicating that these procedures are incredibly durable and should be considered in young, fit patients with complex dissections and genetically triggered aortic diseases. For nondissected aneurysms, patient survival was $63 \%$ at 5 years, which compares favorably with our patient survival of $59 \%$ in a higher-risk group. In octogenarians, Aftab and colleagues ${ }^{28}$ reported significantly higher morbidity and mortality.

Selection of open or endovascular repair should be tailored to patient age, clinical risk, presence of genetically triggered aortic diseases, and anatomy. For patients aged less than 65 years and who have genetically triggered aortic diseases, we continue to offer open surgical repair as the first treatment option. However, we have expanded the indications of endovascular repair to include not only higherrisk patients but also those who are low or intermediate risk and have favorable anatomy. The ideal patient has minimal atherosclerotic debris within healthy aortic segments, adequate iliofemoral and brachial access, and suitable renal and mesenteric target vessels with no early bifurcation or occlusive disease.

\section{Study Limitations}

Although the clinical data on patients treated by manufactured devices were collected prospectively, the experience with PMEGs was retrospective using off-label device modifications to treat a higher-risk group. There was a 
significant impact of the learning curve, which is reflected by significant differences in patient selection (Table E4) and improvements in procedural variables (Table E5). As a result, we observed a significant decline in mortality from $7.5 \%$ to $1.2 \%$ in the second half experience. Our results should not be generalized, and outcomes such as type III endoleaks, branch stenosis, and reinterventions are likely to be affected by improvements in device design and side branch technology. Because our sample size is small, we were not able to analyze outcomes by specific groups among patients with more extensive TAAAs.

\section{CONCLUSIONS}

Endovascular repair of TAAAs continues to evolve with improvements in device design, implantation techniques, and adjunctive maneuvers to decrease mortality and paraplegia. Our study shows high technical success and low mortality and morbidity, which improved in the late experience after introduction of manufactured devices. There was a high rate of reinterventions to treat type III endoleaks and branch vessel stenosis, which should be the focus of future refinements in the technique. Although this technique has major benefits in the elderly and higher-risk patients, long-term data are still needed before widespread use in lower-risk groups.

\section{Conflict of Interest Statement}

G.S.O. has a consulting agreement with Cook Medical Inc, and WL Gore \& Associates (all consulting fees paid to the Mayo Clinic) and receives research grants from Cook Medical Inc, and WL Gore \& Associates (paid to the Mayo Clinic). All other authors have nothing to disclose with regard to commercial support.

\section{References}

1. Chuter TA, Gordon RL, Reilly LM, Goodman JD, Messina LM. An endovascular system for thoracoabdominal aortic aneurysm repair. J Endovasc Ther. 2001;8: 25-33.

2. Fernandez CC, Sobel JD, Gasper WJ, Vartanian SM, Reilly LM, Chuter TAM, et al. Standard off-the-shelf versus custom-made multibranched thoracoabdominal aortic stent grafts. J Vasc Surg. 2016;63:1208-15.

3. Eagleton MJ, Follansbee M, Wolski K, Mastracci T, Kuramochi Y. Fenestrated and branched endovascular aneurysm repair outcomes for type II and III thoracoabdominal aortic aneurysms. J Vasc Surg. 2016;63:930-42.

4. Kasprzak PM, Gallis K, Cucuruz B, Pfister K, Janotta M, Kopp R. Editor's choice-Temporary aneurysm sac perfusion as an adjunct for prevention of spinal cord ischemia after branched endovascular repair of thoracoabdominal aneurysms. Eur J Vasc Endovasc Surg. 2014;48:258-65.

5. Katsargyris A, Oikonomou K, Kouvelos G, Renner H, Ritter W, Verhoeven ELG. Spinal cord ischemia after endovascular repair of thoracoabdominal aortic aneurysms with fenestrated and branched stent grafts. J Vasc Surg. 2015;62:1450-6.

6. Dias NV, Sonesson B, Kristmundsson T, Holm H, Resch T. Short-term outcome of spinal cord ischemia after endovascular repair of thoracoabdominal aortic aneurysms. Eur J Vasc Endovasc Surg. 2015;49:403-9.

7. O'Callaghan A, Mastracci TM, Eagleton MJ. Staged endovascular repair of thoracoabdominal aortic aneurysms limits incidence and severity of spinal cord ischemia. J Vasc Surg. 2015;61:347-54.e1.

8. Maurel B, Delclaux N, Sobocinski J, Hertault A, Martin-Gonzalez T, Moussa M, et al. The impact of early pelvic and lower limb reperfusion and attentive perioperative management on the incidence of spinal cord ischemia during thoracoab- dominal aortic aneurysm endovascular repair. Eur J Vasc Endovasc Surg. 2015 49:248-54.

9. Banga PV, Oderich GS, Reis de Souza L, Hofer J, Cazares Gonzalez ML, Pulido JN, et al. Neuromonitoring, cerebrospinal fluid drainage, and selective use of iliofemoral conduits to minimize risk of spinal cord injury during complex endovascular aortic repair. J Endovasc Ther. 2016;23:139-49.

10. Crawford ES, Crawford JL, Safi HJ, Coselli JS, Hess KR, Brooks B, et al. Thoracoabdominal aortic aneurysms: preoperative and intraoperative factors determining immediate and long-term results of operations in 605 patients. $J$ Vasc Surg. 1986;3:389-404.

11. Fillinger MF, Greenberg RK, McKinsey JF, Chaikof EL. Society for Vascular Surgery Ad Hoc Committee on TEVAR Reporting Standards. Reporting standards for thoracic endovascular aortic repair (TEVAR). J Vasc Surg. 2010;52: 1022-33. 1033.e15.

12. Oderich GS. Technique of adding a diameter-reducing wire to the modified TX2 fenestrated stent graft. Vascular. 2010;18:350-5.

13. Oderich GS, Fatima J, Gloviczki P. Stent graft modification with mini-cuff rein forced fenestrations for urgent repair of thoracoabdominal aortic aneurysms. J Vasc Surg. 2011;54:1522-6.

14. Oderich GS, Mendes BC, Correa MP. Preloaded guidewires to facilitate endovascular repair of thoracoabdominal aortic aneurysm using a physician-modified branched stent graft. J Vasc Surg. 2014;59:1168-73.

15. Kitagawa A, Greenberg RK, Eagleton MJ, Mastracci TM, Roselli EE. Fenestrated and branched endovascular aortic repair for chronic type B aortic dissection with thoracoabdominal aneurysms. J Vasc Surg. 2013;58:625-34.

16. Oikonomou K, Kopp R, Katsargyris A, Pfister K, Verhoeven EL, Kasprzak P. Outcomes of fenestrated/branched endografting in post-dissection thoracoabdominal aortic aneurysms. Eur J Vasc Endovasc Surg. 2014;48:641-8.

17. Martin-Gonzalez T, Mastracci T, Carrell T, Constantinou J, Dias N, Katsagyris A, et al. Mid-term outcomes of renal branches versus renal fenestrations for thoracoabdominal aneurysm repair. Eur J Vasc Endovasc Surg. 2016;52:141-8.

18. Mastracci TM, Greenberg RK, Eagleton MJ, Hernandez AV. Durability of branches in branched and fenestrated endografts. J Vasc Surg. 2013;57:926-33.

19. Sugimoto M, Panuccio G, Bisdas T, Berekoven B, Torsello G, Austermann M Tortuosity is the significant predictive factor for renal branch occlusion after branched endovascular aortic aneurysm repair. Eur J Vasc Endovasc Surg. 2016;51:350-7.

20. Conway BD, Greenberg RK, Mastracci TM, Hernandez AV, Coscas R. Renal artery implantation angles in thoracoabdominal aneurysms and their implications in the era of branched endografts. J Endovasc Ther. 2010;17:380-7.

21. Greenberg RK, Lu Q, Roselli EE, Svensson LG, Moon MC, Hernandez AV, et al. Contemporary analysis of descending thoracic and thoracoabdominal aneurysm repair: a comparison of endovascular and open techniques. Circulation. 2008; 118:808-17.

22. Eagleton MJ, Shah S, Petkosevek D, Mastracci TM, Greenberg RK. Hypogastric and subclavian artery patency affects onset and recovery of spinal cord ischemia associated with aortic endografting. J Vasc Surg. 2014;59:89-94.

23. O'Brien N, D'Elia P, Sobocinski J, Maioli F, d'Utra G, Perot C, et al. Inverted limbs in fenestrated and branched endografts. J Endovasc Ther. 2010;17:624-30.

24. Bischoff MS, Scheumann J, Brenner RM, Ladage D, Bodian CA, Kleinman G, et al. Staged approach prevents spinal cord injury in hybrid surgicalendovascular thoracoabdominal aortic aneurysm repair: an experimental model Ann Thorac Surg. 2011;92:138-46.

25. Etz CD, Debus ES, Mohr FW, Kölbel T. First-in-man endovascular preconditioning of the paraspinal collateral network by segmental artery coil embolization to prevent ischemic spinal cord injury. J Thorac Cardiovasc Surg. 2015;149:1074-9.

26. O'Callaghan A, Greenberg RK, Eagleton MJ, Bena J, Mastracci TM. Type Ia endoleaks after fenestrated and branched endografts may lead to component instability and increased aortic mortality. J Vasc Surg. 2015;61:908-14.

27. Coselli JS, LeMaire SA, Preventza O, de la Cruz KI, Cooley DA, Price MD, et al Outcomes of 3309 thoracoabdominal aortic aneurysm repairs. J Thorac Cardiovasc Surg. 2016;151:1323-38.

28. Aftab M, Songdechakraiwut T, Green SY, Zarda S, Price MD, Nalty CC, et al Contemporary outcomes of open thoracoabdominal aortic aneurysm repair in octogenarians. J Thorac Cardiovasc Surg. 2015;149(2 Suppl):S134-41.

Key Words: fenestrated and branched stent-grafts, thoracoabdominal aortic aneurysms, endovascular repair, fenestrations, branches 


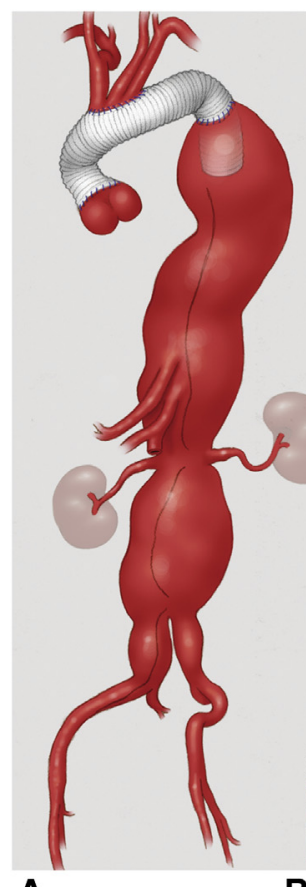

A

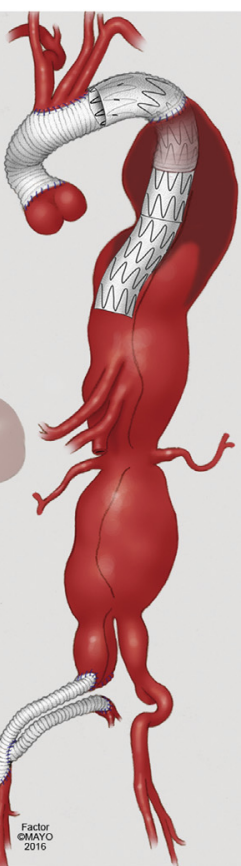

B
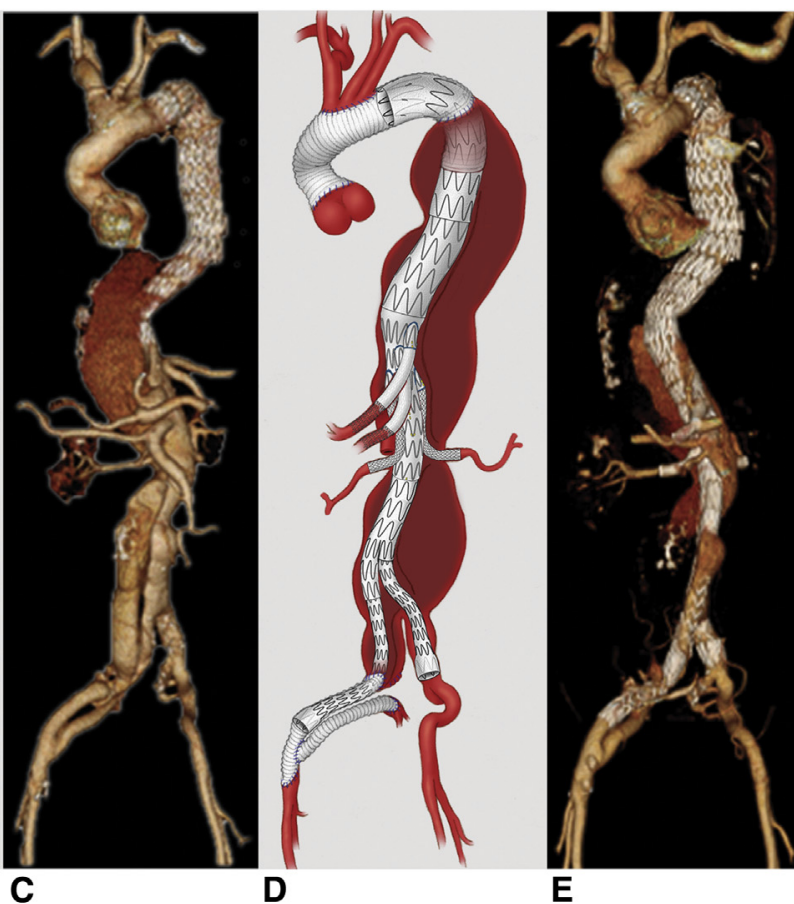

E

FIGURE E1. Illustration of a patient who underwent prior elephant trunk repair for extensive thoracoabdominal dissection (A), followed by first-stage thoracic endovascular aortic repair with right iliac artery reconstruction (B and C). The patient had complete repair in 1 week using a multibranched off-the-shelf stent-graft (D and E).
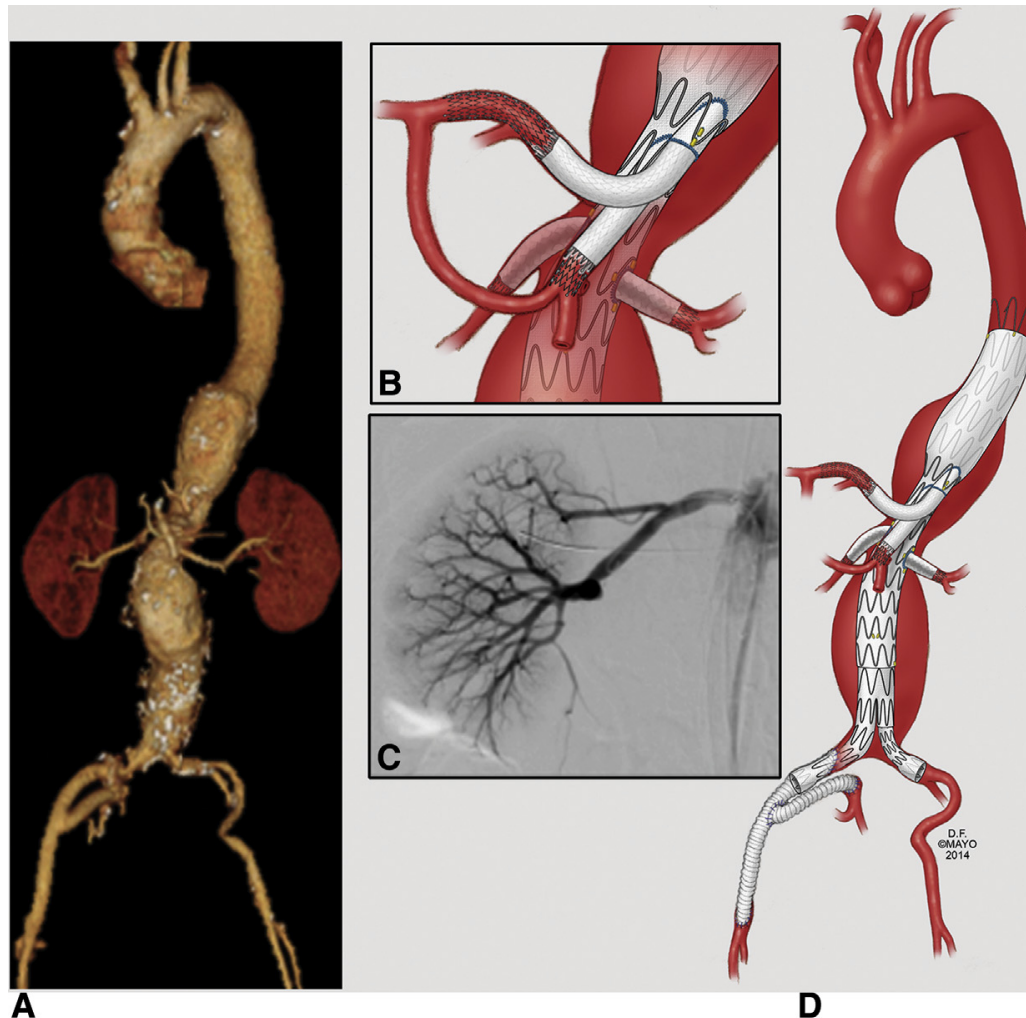

D

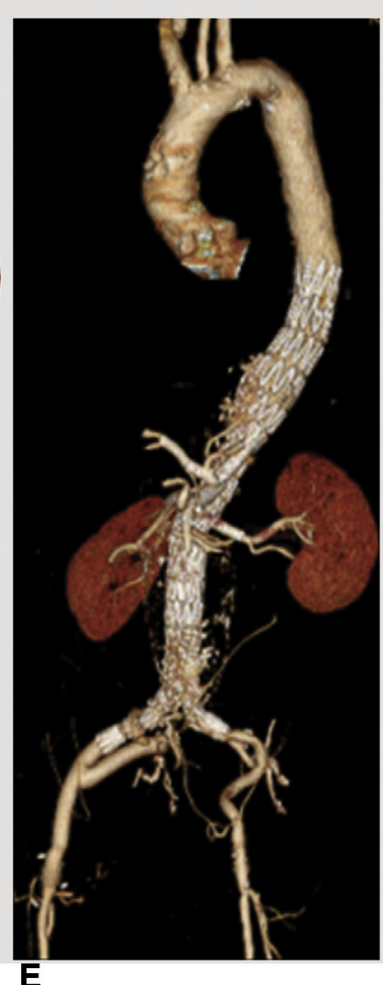

FIGURE E2. Computed tomography angiography of a patient with extent III TAAA (A), treated by patient-specific design using directional branches for the celiac axis and superior mesenteric artery (B) and fenestrations for the renal arteries (C). Illustration and computed tomography angiography of the complete repair (D and $\mathrm{E})$. 


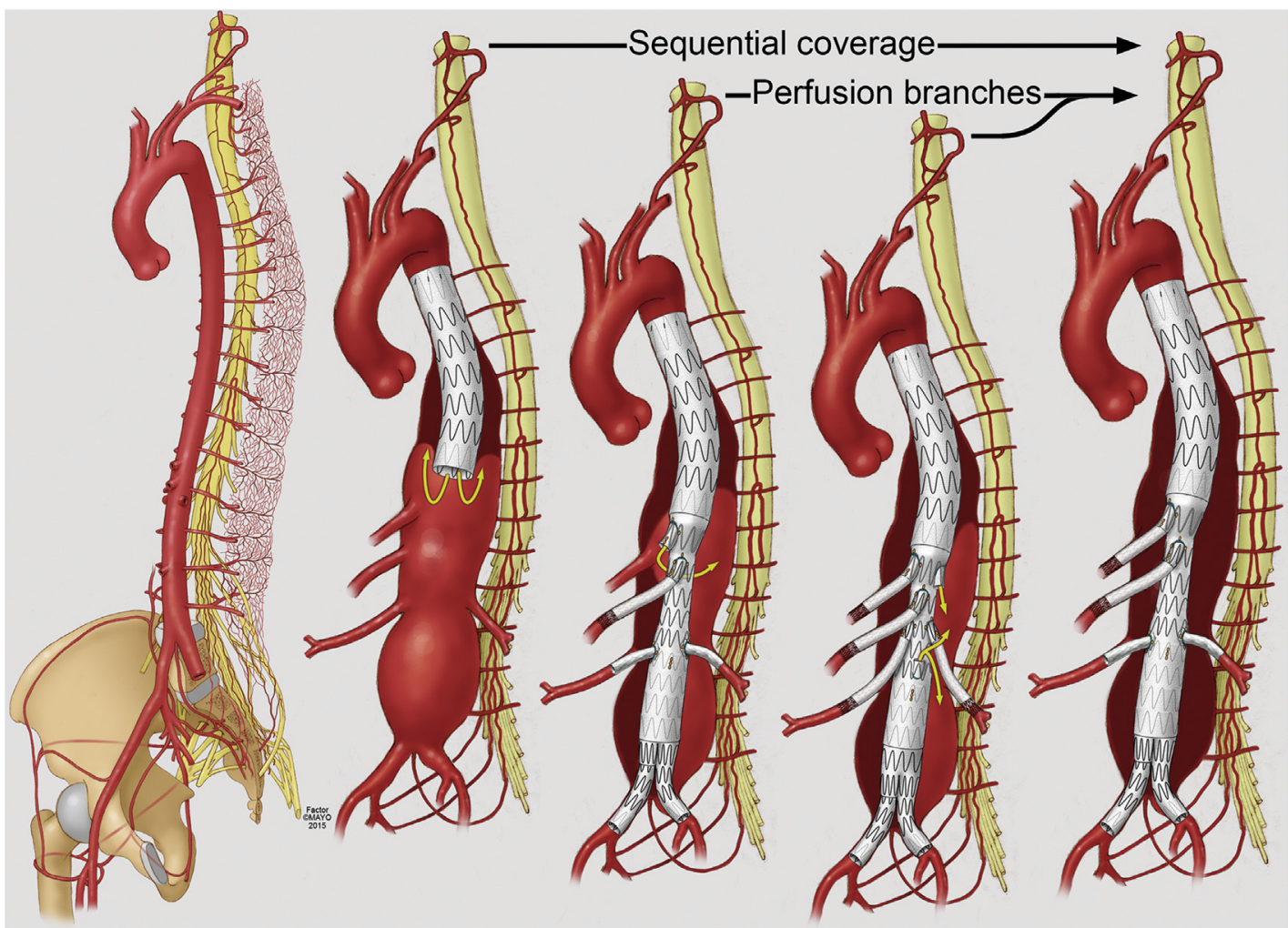

FIGURE E3. Staging of extensive TAAAs can be done using proximal thoracic endovascular repair or perfusion branches. Printed with permission from Banga and colleagues. 


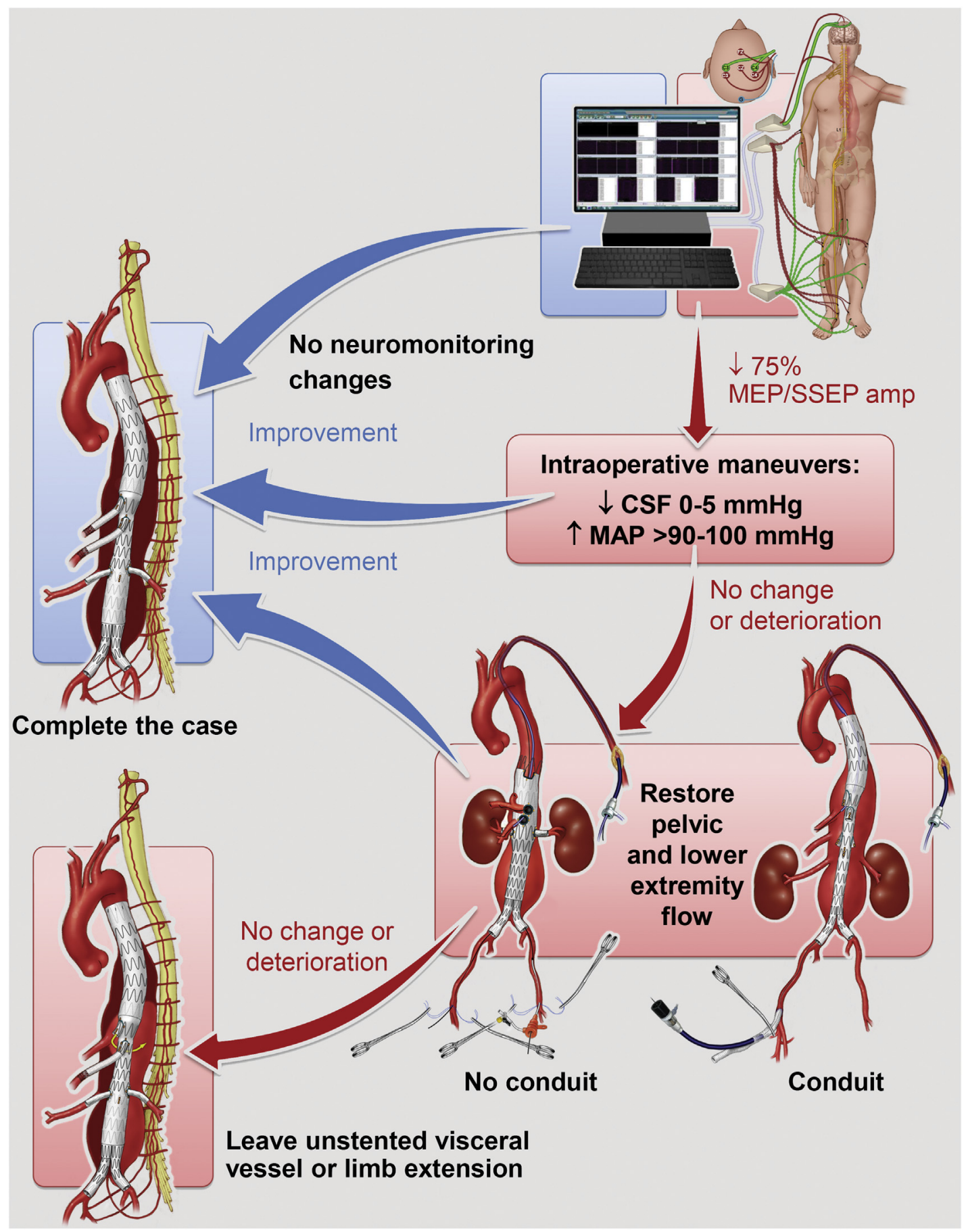

FIGURE E4. Standardized maneuvers and protocol triggered by changes in motor and somatosensory evoked potentials. Printed with permission from Banga and colleagues. ${ }^{9}$ MEP, Motor evoked potential; SSEP, somatosensory evoked potential; CSF, cerebrospinal fluid; MAP, mean arterial pressure. 


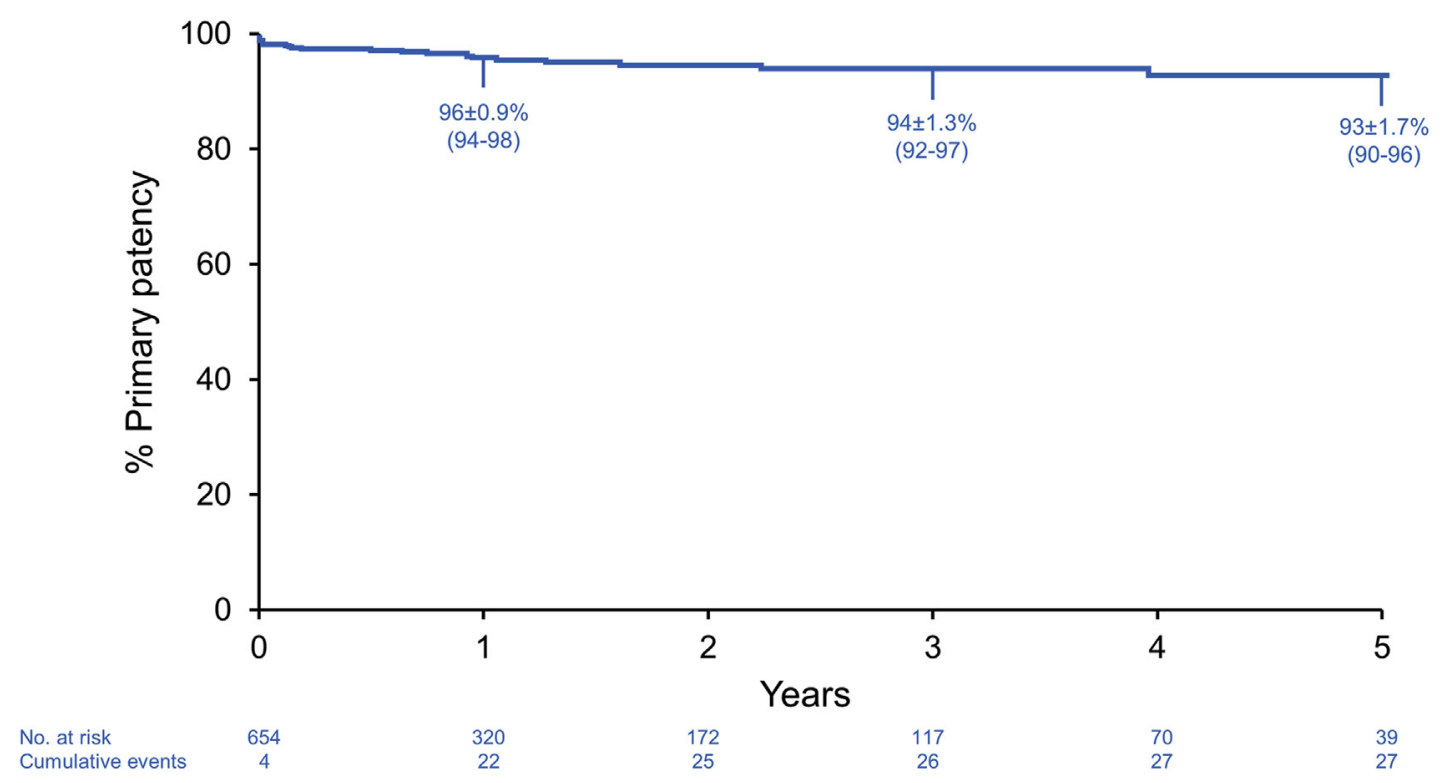

FIGURE E5. Kaplan-Meier estimates of primary target vessel patency in patients treated for TAAAs with 681 renal-mesenteric fenestrations and branches.

TABLE E1. Stent-graft design in 185 patients treated for thoracoabdominal aortic aneurysms with fenestrated-branched stent-grafts

\begin{tabular}{|c|c|c|c|c|c|c|c|}
\hline & Type I-III & & Type IV & & & Overall & \\
\hline Variable & $(\mathbf{N}=73)$ & $\%$ & $(N=112)$ & $\%$ & $P$ value & $(N=185)$ & $\%$ \\
\hline Manufactured grafts & 39 & 53 & 68 & 61 & & 107 & 58 \\
\hline Physician-modified grafts & 34 & 47 & 44 & 39 & & 78 & 42 \\
\hline Celiac axis & & & & & $<.001$ & & \\
\hline No & 9 & 12 & 5 & 5 & & 14 & 8 \\
\hline Fenestration & 27 & 37 & 96 & 86 & & 123 & 67 \\
\hline Directional branch & 37 & 51 & 10 & 9 & & 47 & 26 \\
\hline SMA & & & & & $<.001$ & & \\
\hline No* & 2 & 3 & 0 & 0 & & 2 & 1 \\
\hline Fenestration & 34 & 47 & 103 & 92 & & 137 & 74 \\
\hline Directional branch & 37 & 51 & 9 & 8 & & 46 & 25 \\
\hline Right renal artery & & & & & $<.001$ & & \\
\hline No & 12 & 16 & 11 & 10 & & 23 & 13 \\
\hline Fenestration & 38 & 52 & 96 & 86 & & 134 & 73 \\
\hline Directional branch & 23 & 32 & 4 & 4 & & 27 & 15 \\
\hline Left renal artery & & & & & $<.001$ & & \\
\hline No & 9 & 12 & 8 & 7 & & 17 & 9 \\
\hline Fenestration & 46 & 63 & 98 & 88 & & 144 & 78 \\
\hline Directional branch & 18 & 25 & 5 & 5 & & 23 & 13 \\
\hline
\end{tabular}

$N$, Number of patients; SMA, superior mesenteric artery. *Two patients had celiomesenteric trunks incorporated by single fenestration. 
TABLE E2. Causes of early and late death among patients treated for thoracoabdominal aortic aneurysms

\begin{tabular}{|c|c|c|c|c|c|c|}
\hline & Extent I-III & & Extent IV & & Total & \\
\hline Variable & $\mathbf{N}=\mathbf{7 3}$ & $\%$ & $\mathbf{N}=112$ & $\%$ & $\mathbf{N}=\mathbf{1 8 5}$ & $\%$ \\
\hline Early mortality & 6 & 8 & 2 & 2 & 8 & 4 \\
\hline Acute MI & 0 & 0 & 1 & 1 & 1 & 1 \\
\hline $\begin{array}{l}\text { Multisystem failure due to sepsis (complications } \\
\text { of ischemic colitis) }\end{array}$ & 0 & 0 & 1 & 1 & 1 & 1 \\
\hline Type A dissection & 1 & 1 & 0 & 0 & 1 & 1 \\
\hline Ischemic colitis & 1 & 1 & 0 & 0 & 1 & 1 \\
\hline Cardiac arrest after rapid ventricular pace & 1 & 1 & 0 & 0 & 1 & 1 \\
\hline Acute on chronic renal failure & 1 & 1 & 0 & 0 & 1 & 1 \\
\hline Multifocal stroke/MI & 1 & 1 & 0 & 0 & 1 & 1 \\
\hline Unknown & 1 & 1 & 0 & 0 & 1 & 1 \\
\hline Late mortality & 11 & 15 & 20 & 18 & 31 & 17 \\
\hline Metastatic advanced small cell & 0 & 0 & 1 & 1 & 1 & 1 \\
\hline Respiratory failure/UTI/malnutrition & 0 & 0 & 1 & 1 & 1 & 1 \\
\hline Complications of subdural hematoma & 0 & 0 & 1 & 1 & 1 & 1 \\
\hline Esophageal cancer & 0 & 0 & 1 & 1 & 1 & 1 \\
\hline Congestive heart failure & 0 & 0 & 3 & 3 & 3 & 2 \\
\hline Consumptive syndrome/chronic & 1 & 1 & 0 & 0 & 1 & 1 \\
\hline Mantle cell lymphoma & 1 & 1 & 0 & 0 & 1 & 1 \\
\hline Proximal rupture of the descending thoracic aorta & 1 & 1 & 0 & 0 & 1 & 1 \\
\hline Unknown & 8 & 11 & 13 & 12 & 21 & 11 \\
\hline Total & 17 & 23 & 22 & 20 & 39 & 21 \\
\hline
\end{tabular}

$N$, Number of patients; $M I$, myocardial infarction; UTI, urinary tract infection.

TABLE E3. Summary of types of branch related failures in patients with physician-modified endovascular grafts and manufactured devices

\begin{tabular}{|c|c|c|c|}
\hline Variable & Vessel & Type of incorporation & Type of endograft \\
\hline \multicolumn{4}{|c|}{ Reinterventions for endoleak } \\
\hline Type Ic & LRA/SMA & Branches & MD \\
\hline Type III & LRA & Fenestration & MD \\
\hline Type III & SMA & Fenestration & MD \\
\hline Type III & LRA & Fenestration & MD \\
\hline Type III & CA/SMA/RRA/LRA & Fenestrations & MD \\
\hline Type III & SMA & Fenestration & PMEG \\
\hline Type III & SMA/LRA/RRA & Fenestrations & PMEG \\
\hline Type III & SMA & Fenestration & PMEG \\
\hline Type III & RRA & Fenestration & PMEG \\
\hline Type III & CA/SMA/LRA/RRA & Fenestrations & PMEG \\
\hline Type III & RRA & Fenestration & PMEG \\
\hline \multicolumn{4}{|c|}{ Reinterventions for loss of patency } \\
\hline Occlusion & RRA & Fenestration & PMEG \\
\hline Stenosis & RRA/LRA & Fenestrations & MD \\
\hline Stenosis & RRA & Branch & MD \\
\hline Stenosis & RRA & Fenestration & MD \\
\hline Stenosis & SMA & Fenestration & MD \\
\hline Stenosis & LRA & Fenestration & MD \\
\hline Stenosis & CA/LRA & Fenestrations & PMEG \\
\hline Kinking & SMA & Fenestration & PMEG \\
\hline Kinking & SMA & Fenestration & PMEG \\
\hline Kinking & LRA & Fenestration & PMEG \\
\hline
\end{tabular}

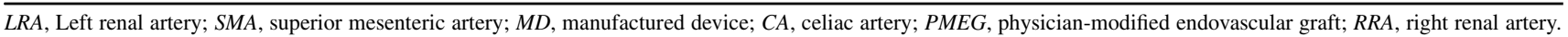


TABLE E4. Demographics and clinical characteristics in 185 patients treated for thoracoabdominal aortic aneurysms with fenestrated-branched stent-grafts, divided in the first and second halves of the surgeon experience

\begin{tabular}{|c|c|c|c|c|c|c|c|}
\hline \multirow{3}{*}{$\begin{array}{ll} & \text { Variable } \\
\text { Age }(y) & \end{array}$} & \multirow{2}{*}{\multicolumn{2}{|c|}{$\begin{array}{c}\text { First half } N=93 \\
\pm \text { SD or } \%\end{array}$}} & \multirow{2}{*}{\multicolumn{2}{|c|}{$\begin{array}{c}\text { Second half } N=92 \\
\pm \text { SD or } \%\end{array}$}} & \multirow{3}{*}{$\frac{P \text { value }}{.56}$} & \multirow{2}{*}{\multicolumn{2}{|c|}{$\begin{array}{c}\text { Overall } \\
(N=185)\end{array}$}} \\
\hline & & & & & & & \\
\hline & 75.01 & \pm 6.72 & 74.41 & \pm 7.29 & & 75 & \pm 7 \\
\hline Male gender & 68 & 73 & 66 & 72 & .83 & 134 & 72 \\
\hline Cardiovascular risk factors & & & & 0 & & & \\
\hline Hypertension & 84 & 90 & 81 & 89 & .77 & 164 & 90 \\
\hline Smoker & 78 & 84 & 82 & 90 & .21 & 159 & 87 \\
\hline Hypercholesterolemia & 77 & 83 & 72 & 79 & .53 & 149 & 81 \\
\hline Coronary artery disease & 66 & 71 & 46 & 51 & .005 & 112 & 61 \\
\hline Chronic obstructive pulmonary disease & 57 & 61 & 32 & 35 & $<.001$ & 88 & 48 \\
\hline MI & 41 & 44 & 27 & 30 & .043 & 68 & 37 \\
\hline Arrhythmia & 25 & 27 & 7 & 8 & $<.001$ & 32 & 17 \\
\hline Diabetes mellitus & 13 & 14 & 18 & 20 & .29 & 31 & 17 \\
\hline $\mathrm{CHF}$ & 17 & 18 & 9 & 10 & .1 & 26 & 14 \\
\hline Stroke/TIA & 9 & 10 & 11 & 12 & .6 & 20 & 11 \\
\hline \multicolumn{8}{|c|}{ Preoperative evaluation and comorbidity scores } \\
\hline Positive cardiac stress test & 24 & 30 & 13 & 15 & .04 & 37 & 23 \\
\hline Baseline creatinine (mg/dL) & 1.34 & \pm 1.08 & 1.28 & \pm 0.80 & .7 & 1.3 & \pm 1 \\
\hline $\begin{array}{l}\text { Baseline glomerular filtration rate } \\
\left(\mathrm{mL} / \mathrm{min} / 1.73 \mathrm{~m}^{2}\right)\end{array}$ & 61.04 & \pm 21.80 & 59.85 & \pm 19.82 & .7 & 60 & \pm 21 \\
\hline Chronic kidney disease stage III to $\mathrm{V}$ & & 0 & & 0 & .013 & 60 & 32 \\
\hline Stage III & 35 & 83 & 14 & 74 & & 49 & 82 \\
\hline Stage IV & 3 & 7 & 5 & 26 & & 8 & 13 \\
\hline Stage V & 3 & 7 & 0 & 0 & & 3 & \\
\hline BMI $\left(\mathrm{kg} / \mathrm{m}^{2}\right)$ & 28.02 & \pm 6.33 & 28.16 & \pm 5.16 & .87 & 28 & \pm 6 \\
\hline ASA clinical score & & & & & $<.001$ & & \\
\hline I & 7 & 8 & 9 & 10 & & 16 & \\
\hline II & 32 & 34 & 60 & 65 & & 91 & 49 \\
\hline III & 43 & 46 & 18 & 20 & & 61 & 33 \\
\hline IV & 11 & 12 & 2 & 2 & & 13 & \\
\hline $\mathrm{V}$ & 0 & 0 & 3 & 3 & & 3 & 2 \\
\hline SVS total score $(0-30)$ & 1.35 & \pm 0.44 & 1.19 & \pm 0.40 & .013 & 13 & \pm 4 \\
\hline Connective tissue disorder & 3 & 3 & 1 & 1 & .24 & 4 & 2 \\
\hline Peripheral arterial disease & 36 & 39 & 24 & 26 & .07 & 60 & 33 \\
\hline \multicolumn{8}{|l|}{ Medical history } \\
\hline Previous aortic surgery & 34 & 37 & 35 & 38 & .83 & 69 & 38 \\
\hline Previous abdominal surgery & 45 & 48 & 43 & 47 & .82 & 87 & 47 \\
\hline Endovascular aortic intervention & 13 & 14 & 22 & 24 & .08 & 35 & 19 \\
\hline History of malignancy & 26 & 28 & 18 & 20 & .19 & 43 & 23 \\
\hline Family history of aortic aneurysm & 4 & 100 & 12 & 80 & .33 & 20 & 13 \\
\hline Hostile abdomen & 39 & 42 & 18 & 20 & $<.001$ & 57 & 31 \\
\hline
\end{tabular}

Continuous data are presented as mean $\pm \mathrm{SD}$; categoric data are given in percentage. $N$, Number of patients; $S D$, standard deviation; $M I$, myocardial infarction; $C H F$, congestive heart failure; TIA, transient ischemic attack; BMI, body mass index; ASA, American Society of Anesthesiologists; SVS, Society for Vascular Surgery. 
TABLE E5. Procedural variables and stent design in 185 patients treated for thoracoabdominal aortic aneurysms with fenestrated-branched stentgrafts, divided in the first and second halves of the surgeon experience

\begin{tabular}{|c|c|c|c|c|c|}
\hline & First half & & Second half & & \\
\hline Variable & $\mathbf{N}=\mathbf{9 3}$ & $\%$ or mean \pm SD & $\mathbf{N}=92$ & $\%$ or mean \pm SD & $P$ value \\
\hline General anesthesia & 93 & $100 \%$ & 91 & $99 \%$ & .31 \\
\hline Cerebrospinal fluid drainage & 69 & $74 \%$ & 78 & $85 \%$ & .07 \\
\hline SSP/motor-evoked potentials & 31 & $33 \%$ & 79 & $86 \%$ & $<.001$ \\
\hline Percutaneous & 27 & $29 \%$ & 69 & $76 \%$ & $<.001$ \\
\hline Amount of contrast used (mL) & 195 & \pm 83.08 & 170.1 & \pm 55.77 & .016 \\
\hline Total fluoroscopy time (min) & 109 & \pm 49.99 & 80.14 & \pm 30.00 & $<.001$ \\
\hline Estimated blood loss (mL) & 1268 & \pm 1457.92 & 533.8 & \pm 564.21 & $<.001$ \\
\hline \multicolumn{6}{|l|}{ Transfusion of blood products } \\
\hline PRBC & 1208 & \pm 1080.94 & 702.5 & \pm 481.26 & .015 \\
\hline FFP & 863 & \pm 604.39 & 726.9 & \pm 375.83 & .55 \\
\hline Platelets & 539 & \pm 278.38 & 371 & \pm 183.44 & .15 \\
\hline Cryoprecipitate & 206 & \pm 11.59 & 197 & \pm 66.68 & .76 \\
\hline Device & & & & & $<.001$ \\
\hline Manufactured grafts & 19 & $20 \%$ & 88 & $96 \%$ & \\
\hline Physician modified & 74 & $80 \%$ & 4 & $4 \%$ & \\
\hline Device type & & & & & $<.001$ \\
\hline IDE & 18 & $19 \%$ & 88 & $96 \%$ & \\
\hline Z-fen & 1 & $1 \%$ & 0 & $0 \%$ & \\
\hline Physician modified & 74 & $80 \%$ & 4 & $4 \%$ & \\
\hline Total renal mesenteric vessels $\geq 4$ target vessels & 65 & $70 \%$ & 76 & $83 \%$ & .042 \\
\hline Total renal-mesenteric vessels & 3.58 & \pm 0.78 & 3.8 & \pm 0.77 & .05 \\
\hline No. of fenestrations & 3.39 & \pm 1.09 & 2.43 & \pm 1.71 & $<.001$ \\
\hline No. of scallops & 0.03 & \pm 0.18 & 0 & \pm 0.00 & .09 \\
\hline No. of directional branches & 0.16 & \pm 0.66 & 1.45 & \pm 1.63 & $<.001$ \\
\hline No. of perfusion branches & 0.03 & \pm 0.18 & 0 & \pm 0.00 & .09 \\
\hline Conduit & 24 & $27 \%$ & 24 & $26 \%$ & .89 \\
\hline
\end{tabular}

